\title{
Real-Time Speckle Reduction and Coherence Enhancement in Ultrasound Imaging via Nonlinear Anisotropic Diffusion
}

\author{
Khaled Z. Abd-Elmoniem, Student Member, IEEE, Abou-Bakr M. Youssef, and Yasser M. Kadah*, Member, IEEE
}

\begin{abstract}
This paper presents a novel approach for speckle reduction and coherence enhancement of ultrasound images based on nonlinear coherent diffusion (NCD) model. The proposed NCD model combines three different models. According to speckle extent and image anisotropy, the NCD model changes progressively from isotropic diffusion through anisotropic coherent diffusion to, finally, mean curvature motion. This structure maximally low-pass filters those parts of the image that correspond to fully developed speckle, while substantially preserving information associated with resolved-object structures. The proposed implementation algorithm utilizes an efficient discretization scheme that allows for real-time implementation on commercial systems. The theory and implementation of the new technique are presented and verified using phantom and clinical ultrasound images. In addition, the results from previous techniques are compared with the new method to demonstrate its performance.
\end{abstract}

Index Terms-Coherence enhancement, nonlinear anisotropic diffusion, speckle reduction, ultrasound imaging.

\section{INTRODUCTION}

$\mathbf{T}$ HE noninvasive nature, low cost, portability, and real-time image formation make ultrasound imaging an essential tool for medical diagnosis. Over the years, its application extended to include many fields and research is underway to improve the technology even further. One of the areas where research in this field has addressed is the fundamental problem of speckle noise, which is a major limitation on image quality in ultrasound imaging. The presence of speckle noise affects human interpretation of the images as well as the accuracy of computer-assisted diagnostic techniques. Poor image quality in some cases makes feature extraction, analysis, recognition, and quantitative measurements impossible.

A number of methods have been proposed to address the problem of speckle noise including temporal averaging, median filtering, maximum amplitude writing (temporal dilation), adaptive speckle reduction (ASR) (statistical enhancement), homomorphic Wiener filtering, and wavelet shrinkage (WS). Temporal averaging and multiframe methods try to increase

Manuscript received September 21, 2001; revised May 3, 2002. This work was supported in part by the R\&D Department at International Biomedical Engineering (IBE Technologies), Giza, Egypt. Asterisk indicates corresponding author.

K. Z. Abd-Elmoniem and A.-B. M. Youssef are with the Biomedical Engineering Department, Cairo University, Giza 12613, Egypt.

*Y. M. Kadah is with the Biomedical Engineering Department, Cairo University, Giza 12613, Egypt (e-mail: ymk@ieee.org).

Publisher Item Identifier 10.1109/TBME.2002.802051. the signal-to-noise ratio (SNR) by generating multiple uncorrelated images that are summed incoherently to reduce speckle. Despite being simple and fast, these approaches suffer from two limitations. First, in order to produce uncorrelated images, the transducer has to be translated at least by about half its element width for each of the generated frames [1]. Second, temporal averaging based on transducer movement causes the loss of small details such as small vessels and texture patterns because of blurring.

A more reliable technique based on ASR filtering was proposed in [3]-[7]. This technique depends on the SNR and possibly the autocorrelation function to permit a varying degree of smoothing according to the extent of the speckle pattern from the fully formed speckle (FFS), which is known to follow a Rayleigh distribution. This approach works well when applied to the uncompressed backscattered envelope signal but becomes severely inaccurate with the log-compressed signal. Moreover, the parameters introduced in this method (such as neighborhood, structure, and speckle thresholds) may not correlate well with actual speckle models.

In another method based on adaptive weighted median filter (AWMF) [8], pixel values are replaced by the weighted median of a local neighborhood whose width is determined based on the SNR around that pixel. The over simplification of this method led to limited success of this method given that fine image details are usually lost.

Speckle reduction via filtering in the wavelet domain was proposed by several groups. Most of these techniques are based on the idea of soft-thresholding denoising first presented by Donoho [22]. In this method, the signal is decomposed in the wavelet domain and the obtained wavelet coefficients are then soft-thresholded. That is, the wavelet coefficients with absolute values below a certain threshold are replaced by zero, while those above it are only modified by shrinking them toward zero. A modification of this technique is to apply nonlinear soft thresholding within finer levels of scales to suppress speckle [17]. Feature enhancement was accomplished via stretching of wavelet coefficients in the midrange levels. The estimated image is reconstructed by taking the inverse wavelet transform of the processed coefficients.

Since the above methods could not succeed to balance between speckle suppression and feature preservation due to the complexity of speckle statistics, another technique was proposed to process the signal before it is logarithmically compressed for its simple characteristics [15]. First, the recorded image is low-pass filtered. Then, the filtered part and 
the discarded part are decomposed by wavelet transform. The coefficients of both parts pass through soft-thresholding and finally merge to construct the final speckle-suppressed image.

In spite of the encouraging results obtained using the above techniques, they have not been widely considered for practical implementation because of their high computational complexity and large memory space requirements. Furthermore, some techniques require the signal be processed before logarithmic compression [23], which is not usually possible in most commercial ultrasound systems. In addition, the stability and robustness of such techniques against parameter variations have not been addressed. Therefore, a technique that relies on a more accurate model for speckle noise while maintaining low complexity, simple implementation, and robustness against parameter variations would be rather valuable for practical use.

In this paper, we describe a new speckle reduction technique whereby the log-compressed ultrasound pulse-echo image is smoothed to suppress the FFS while substantially preserving image components corresponding to resolved (or partially resolved) object structures. This technique is based on a nonlinear diffusion model adapted to remove the compressed speckle pattern from the raw lines acquired using a convex array ultrasound imager (without loss of generality). We demonstrate that the use of raw lines instead of the formed image is more accurate since the actual scan is rather in radial coordinates than in Cartesian coordinates and that it is nearly an order of magnitude faster than when applied to the formed image. Due to its anisotropy, the proposed technique allows coherent structure enhancement while the dynamics of the proposed diffusion model is controlled by the local behavior of the signal. The model measures signal coherence, which is an important speckle feature, to control the direction and magnitude of diffusion. A modified version of this model is also proposed to ensure parameter robustness of the technique whereby a bias term is added in such a way not to impose significant additional computational effort. This bias term enables the technique to reach a meaningful steady-state that is different from the constant trivial solution. To ensure real-time implementation, a special discretization scheme is proposed to further reduce the complexity of this method. The stability and robustness of the new techniques are analyzed and verified by experiments using computer simulations as well as actual ultrasound images.

\section{SPECKLE MODEL}

\section{A. Medical Ultrasound Speckle Pattern}

The nature of the speckle pattern can be categorized into one of three classes according to the number of scatterers per resolution cell or the so called scatterer number density (SND), in addition to their spatial distribution and the characteristics of the imaging system itself. These classes are described as follows:

1) FFS pattern, which occurs when many fine randomly distributed scattering sites exist within the resolution cell of the pulse-echo system $(\mathrm{SND}>10)$. In this case, the amplitude of the backscattered signal can be modeled as a Rayleigh distributed random variable with a constant SNR of 1.92. Under such conditions, the textural features of the speckle pattern represent a multivariate signature of the imaging instrument and its point spread function [3]. Blood cells are typical examples of this type of scatterers.

2) Nonrandomly distributed with long-range order (NRLR) [2]. Examples of this type are the lobules in liver parenchyma. It contributes a coherent or specular backscattered intensity that is in itself spatially varying. Due to the correlation between scatterers, the effective number of scatterers is finite (SND $<10$ ). This situation can be modeled by the K-distribution. This type is associated with SNR below 1.92 [15]. It can also be modeled by the Nakagami distribution [29].

3) Nonrandomly distributed with short-range order (NRSR) [2]. Examples of this type include organ surfaces and blood vessels. When a spatially invariant coherent structure is present within the random scatterer region, the probability density function (PDF) of the backscattered signals becomes close to the Rician distribution. This class is associated with SNR above 1.92 [15].

Hence, the coherence phenomena make the SNR an ambiguous feature that cannot be used alone to characterize the speckle model. The deviation in image properties due to the presence of coherent structures that are partially or completely resolved results in a speckle pattern that is no longer entirely characteristic of the imaging system [3]. It should, therefore, be possible to use these deviations to classify each local region of the image according to how much it resembles the FFS normally generated by that particular imaging system in that part of the image. This measure of similarity can then be used to control the spatial bandwidth of a smoothing filter of some kind so that regions within the image that closely resemble the FFS are replaced by a local mean value. At the other extreme, regions with properties that are least similar to FFS should be kept unaltered [3].

\section{B. Effect of Logarithmic Compression}

Due to the limited dynamic range of commercial display monitors, ultrasound imaging systems compress the echo signal to fit in the display range. Such compression changes the characteristics of the signal PDF. In particular, it affects the high intensity tail of the Rayleigh and Rician PDFs more than the low intensity part. As a result, the speckle noise becomes very close to white Gaussian noise corresponding the uncompressed Rayleigh signal [15]. It should be noted that some authors suggested the use of a generalization of the Gaussian distribution, namely, the alpha-stable distribution, to model such noise and handle the data with a degree of nonlinearity that corresponds to the deviation from the Gaussian model [27]. In fact, the Gaussian distribution is the limiting case of such class of probability density functions. This approach has an advantage in image areas where very few scatterers are present and the SNR is very poor such that the assumption of Gaussianity is severely violated. This is not a common situation in most sonographic applications though and the associated complexity to use such model may not be justified.

At the output of the beamformer on the ultrasound imaging system and prior to the logarithmic compression stage for the 
envelope signal, speckle noise can be approximated as a multiplicative noise such that

$$
f(x, y)=g(x, y) \eta_{m}(x, y)+\eta_{a}(x, y) .
$$

Here, $\boldsymbol{g}(x, y)$ is an unknown piecewise constant two-dimensional (2-D) function representing the noise-free original image, $f(x, y)$ is the noisy observation of $\boldsymbol{g}(x, y), \eta_{m}$ and $\eta_{a}$ are multiplicative and additive noise, respectively, and $x$ and $y$ are variables of spatial locations that belong to 2-D space of all real numbers, $(x, y) \in \Re^{2}$. Since the effect of additive noise (such as sensor noise) is considerably small compared with that of multiplicative noise (coherent interference) $\left(\left\|\eta_{a}(x, y)\right\|^{2} \ll\left\|\eta_{m}(x, y)\right\|^{2}\right)$, (1) can be rewritten as

$$
f(x, y) \approx g(x, y) \eta_{m}(x, y) \text {. }
$$

The logarithmic amplification transforms the model in (2) into the classical signal in additive noise form. That is

$$
\log (f(x, y))=\log (g(x, y))+\log \left(\eta_{m}(x, y)\right)
$$

or

$$
f^{l}(x, y)=g^{l}(x, y)+\eta_{a}^{l}(x, y) .
$$

Here, $\eta_{a}^{l}(x, y)$ is approximated as additive white noise. We assume here that the speckle pattern has a white Gaussian noise model. This assumption is valid especially if we consider the speckle nature (initially Rayleigh scattered) after envelope detection and logarithmic amplification of the radio-frequency (RF) signal. In the absence of underlying structures, the speckle pattern and noise can both be assumed to have a white Gaussian noise model. It should be noted however that even though this assumption might not hold in the presence of nonflat structures, it still considered close enough for practical purposes.

\section{DifFUSION FILTERING MODELS}

The SNR remains the most fundamental feature that is most widely used in single feature texture classifiers due to its sensitivity to the variation of scatterer distribution in a region. The unsharp filter described in [3] allows the degree of smoothing to be controlled by the local features of image texture. The proposed filter, named ASR, attempts to differentiate between FFS and NRLR classes of speckle. Hence, this filter has the form

$$
\hat{I}=\bar{I}+k(I-\bar{I})
$$

where $\hat{I}$ is the new (processed) value of a pixel to be computed from the old unprocessed value, $I$, and the local mean of the old values surrounding and including that pixel, $\bar{I}$. The constant, $k$, is controlled by the measure of similarity used, $p$, which in this case is the deviation in the ratio of the local variance of gray levels to the local mean [3], [7]. That is

$$
k=\frac{p-\bar{p}_{s}}{p}
$$

and,

$$
p=\frac{\operatorname{var}(I)}{\bar{I}}
$$

or alternatively

$$
p=\left(\frac{1}{\mathrm{SNR}}\right)^{2}+1=\frac{\left\langle I^{2}\right\rangle}{\langle I\rangle^{2}}
$$

where $\bar{p}_{s}$ is the mean value of $p$ in a region corresponding to FFS [3].

\section{A. Nonlinear Anisotropic Diffusion}

Diffusion algorithms remove noise from an image by modifying the image via solving a partial differential equation (PDE). For example, consider applying the isotropic diffusion equation (the heat equation) given by $\partial I(x, y, t) / \partial t=\operatorname{div}(c \nabla I)$, using the original (degraded or noisy) image $I(x, y, 0)$ as the initial condition, where $I(x, y, 0): \Re^{2} \rightarrow \Re^{+}$is an image in the continuous domain, $(x, y)$ specifies spatial position, $t$ is an artificial time parameter, $c$ is the diffusion constant, and where $\nabla I$ is the image gradient. Modifying the image according to this linear isotropic diffusion equation is equivalent to filtering the image with a Gaussian filter.

The previous ASR technique can be expressed in the form

$$
\hat{I}-I=(1-k)(\bar{I}-I)
$$

or,

$$
\hat{I}_{t+1}-I_{t}=(1-k)\left(\bar{I}_{t}-I_{t}\right)
$$

which is very close to the nonlinear isotropic diffusion model described by

$$
I_{t+1}-I_{t}=g(p)\left(I_{\sigma x x}+I_{\sigma y y}\right) .
$$

Here, $I_{\sigma}$ is the ultrasound image after smoothing with a linear Gaussian kernel of scale $\sigma, I_{\sigma x x}$ and $I_{\sigma y y}$ are the Laplacian of the smoothed image in the $x$ and $y$ directions, respectively, $g(p)$ is a function of the SNR or $g=\bar{p}_{s} / p$, which represents a nonlinear diffusivity replacing the constant diffusivity in the linear case. Diffusion is maximum (i.e., $g(p)=1$ ) in a Rayleigh scatterer region and zero in a fully structured or correlated region (specified experimentally for each imaging system environment). Simple speckle reduction algorithms like the model above enhance only those regions associated with low SNR, i.e., belonging to NRLR speckle model, while organ surfaces are misclassified and possibly degraded.

\section{B. Perona and Malik Formulation}

In their seminal work, Perona and Malik replaced the classical isotropic diffusion with [9]

$$
\frac{\partial I(x, y, t)}{\partial t}=\operatorname{div}[g(\|\nabla I\|) \cdot \nabla I]
$$

where $\|\nabla I\|$ is the gradient, and $g(\|\nabla I\|)$ is the diffusivity function or the edge-stopping function [13]. This function is chosen such that $g(x) \rightarrow 0$ as $x \rightarrow \infty$, and should be monotonically decreasing so that the diffusion or the smoothing decreases as the gradient strength increases and the diffusion is stopped across edges.

\section{Coherent Nonlinear Anisotropic Diffusion}

Although $g(x)$ can be a scalar function and diffusion is still anisotropic and since $\nabla I$ serves only as an edge detector, the applicability of the above filter is restricted to smoothing with edge enhancement. In general, $g(x)$ can be put into a tensor form that measures local coherence of structures such that the diffusion process becomes more directional in both the gradient and the contour directions, which represent the directions of maximum 
and minimum variations, respectively. Hence, the coherent diffusion model takes the form

$$
\frac{\partial I(x, y, t)}{\partial t}=\operatorname{div}[D \nabla I]
$$

where $D \in \Re^{2 \times 2}$ is a symmetric positive semi-definite diffusion tensor representing the required diffusion in both gradient and contour directions and, hence, enhancing coherent structures as well as edges. The design of $D$ requires estimating the local coherence, which can be represented by the local contour and gradient principal directions and their relative contrast. There are two tensors widely used to detect the local coherence, namely, the structure tensor (also called scatter matrix or windowed second moment tensor) and the Hessian tensor, which represents the second order derivatives. These can be expressed as follows:

$$
\underbrace{\left(\begin{array}{cc}
I_{x}^{2} & I_{x} I_{y} \\
I_{x} I_{y} & I_{y}^{2}
\end{array}\right)}_{\text {Structure matrix }} \underbrace{\left(\begin{array}{ll}
I_{x x} & I_{x y} \\
I_{x y} & I_{y y}
\end{array}\right)}_{\text {Covariance (Hessian) matrix }} .
$$

Because the Hessian matrix is more sensitive to noise, the use of the structure tensor was favored. The multiscale structure matrix takes the form

$$
\begin{aligned}
J_{\rho}(\nabla I) & =K_{\rho} *(\nabla I \otimes \nabla I) \\
& =K_{\rho} *\left(\nabla I \cdot \nabla I^{T}\right) \quad(\rho \geq 0)
\end{aligned}
$$

or equivalently

$$
\begin{aligned}
J_{\rho}(I) & =\left(\begin{array}{cc}
K_{\rho} * I_{x}^{2} & K_{\rho} *\left(I_{x} I_{y}\right) \\
K_{\rho} *\left(I_{x} I_{y}\right) & K_{\rho} * I_{y}^{2}
\end{array}\right) \\
& =\left(\begin{array}{ll}
j_{11} & j_{12} \\
j_{12} & j_{22}
\end{array}\right) .
\end{aligned}
$$

Here, the symbol ' $*$ ' stands for convolution and the convolution kernel is expressed as

$$
K_{\sigma}(x, y)=\left(2 \pi \sigma^{2}\right)^{-1} \cdot \exp \left(\frac{x^{2}+y^{2}}{2 \sigma^{2}}\right) .
$$

The above convolution is done component-wise mainly to average a feature over a known neighborhood (scale) where $\sigma$ is the integration scale (the window size) over which the orientation information is averaged [14]. Using eigenvalue decomposition, the formulation in (10) can be put as

$$
J(I)=\left(\begin{array}{ll}
w_{1} & w_{2}
\end{array}\right)\left(\begin{array}{cc}
\mu_{1} & 0 \\
0 & \mu_{2}
\end{array}\right)\left(\begin{array}{l}
w_{1}^{T} \\
w_{2}^{T}
\end{array}\right) .
$$

Here, the eigenvectors $w_{1}, w_{2}$ and the eigenvalues $\mu_{1}, \mu_{2}$ correspond to the directions of maximum and minimum variations and the strength of these variations, respectively.

\section{Mean Curvature Motion (MCM)}

In general, the above models make smoothing (motion) along the direction of local curvature. On the other hand, no smoothing occurs in the gradient direction. Linear diffusion model causes smoothing isotropically in all directions and it is better expressed as

$$
\frac{\partial \boldsymbol{I}}{\partial t}=\operatorname{div}(\nabla \boldsymbol{I})=\boldsymbol{I}_{\xi \xi}+\boldsymbol{I}_{\eta \eta} .
$$

Here, $\eta$ is the gradient direction; the direction of maximum variation, and $\xi$ is the contour direction; the direction of minimum variation. This model modifies the diffusion equation such that smoothing is now in the $\xi$ direction [10]; the direction of the piecewise stationary process. That is, smoothing with unity diffusivity perpendicular to gradient direction. This can be expressed as [28]

$$
\begin{aligned}
& \frac{\partial I}{\partial t}=\frac{1}{\|\nabla I\|} \operatorname{div}\left(\left(\begin{array}{c}
I_{y} \\
-I_{x}
\end{array}\right)\left(I_{y}-I_{x}\right) \nabla \bar{I}\right) \\
& =\frac{1}{\|\nabla I\|} \operatorname{div}\left(\left(\begin{array}{cc}
I_{y}^{2} & -I_{x} I_{y} \\
-I_{x} I_{y} & I_{x}^{2}
\end{array}\right) \nabla \bar{I}\right) \\
& =\frac{1}{\|\nabla I\|}\left(\begin{array}{ll}
\frac{\partial}{\partial x} & \frac{\partial}{\partial y}
\end{array}\right)\left(\begin{array}{c}
I_{y}^{2} \bar{I}_{x}-I_{x} I_{y} \bar{I}_{y} \\
-I_{x} I_{y} \bar{I}_{x}+I_{x}^{2} \bar{I}_{y}
\end{array}\right) \\
& =\frac{1}{\left(I_{x}^{2}+I_{y}^{2}\right)}\left[\frac{\partial}{\partial x}\left(I_{y}^{2} \bar{I}_{x}-I_{x} I_{y} \bar{I}_{y}\right)\right. \\
& \left.+\frac{\partial}{\partial y}\left(-I_{x} I_{y} \bar{I}_{x}+I_{x}^{2} \bar{I}_{y}\right)\right] \\
& =\frac{I_{y}^{2} I_{x x}-2 I_{x} I_{y} I_{x y}+I_{x}^{2} I_{y y}}{\left(I_{x}^{2}+I_{y}^{2}\right)}=I_{\xi \xi \xi} .
\end{aligned}
$$

Here, a bar was placed on the symbols standing for functions as opposed to the constants in the diffusivity matrix. A modified version has appeared with a monotonically decreasing diffusivity in contour direction only [11]. Another modification has been introduced that adds isotropic term to the equation above [12]. It now takes the form

$$
\frac{\partial I}{\partial t}=\frac{\Delta I+k^{2}\left(I_{y}^{2} I_{x x}-2 I_{x} I_{y} I_{x y}+I_{x}^{2} I_{y y}\right)}{\left(1+k^{2}\|\Delta I\|^{2}\right)^{2}} .
$$

Choosing a large value of $k$ amplifies edges in the image and yields a filter with very strong edge preserving properties. Alternatively, choosing a small value will yield a faster isotropic diffusion. The word faster here refers to the speed of the iterative algorithm to reach the final steady-state, which is the trivial constant solution. That is, the solution as the artificial time $t$ goes to infinity. This can be expressed as

$$
\begin{aligned}
k \rightarrow 0: \boldsymbol{I}_{t} & \rightarrow \Delta \boldsymbol{I} \\
k \rightarrow \infty: \boldsymbol{I}_{t} & \rightarrow \frac{\boldsymbol{I}_{x}^{2} \boldsymbol{I}_{x x}-2 \boldsymbol{I}_{x} \boldsymbol{I}_{y} \boldsymbol{I}_{x y}+\boldsymbol{I}_{y}^{2} \boldsymbol{I}_{y y}}{\left(k\|\Delta \boldsymbol{I}\|^{2}\right)^{2}} \\
& =\frac{1}{k^{2}\|\Delta \boldsymbol{I}\|^{2}} \boldsymbol{I}_{\zeta \zeta} .
\end{aligned}
$$

\section{Nonlinear COHERENT DifFusion (NCD) MODEL}

\section{A. Proposed Model Description}

The diffusion tensor $D$ should be chosen with the same eigenvectors of the structure matrix but with eigenvalues that represent the strength of diffusion in each principal direction. That is

$$
D(I)=\left(\begin{array}{ll}
w_{1} & w_{2}
\end{array}\right)\left(\begin{array}{cc}
\lambda_{1} & 0 \\
0 & \lambda_{2}
\end{array}\right)\left(\begin{array}{l}
w_{1}^{T} \\
w_{2}^{T}
\end{array}\right)
$$

and

$$
\begin{aligned}
& \lambda_{1}= \begin{cases}\alpha \cdot\left(1-\frac{\left(\mu_{1}-\mu_{2}\right)^{2}}{s^{2}}\right), & \text { if }\left(\lambda_{1}-\lambda_{2}\right)^{2} \leq s^{2} \\
0, & \text { else }\end{cases} \\
& \lambda_{2}=\alpha .
\end{aligned}
$$

\section{B. Model Analysis}

The dynamics of the proposed NCD model can be understood from the eigen-analysis framework. The flow at each point is affected by the local coherence or anisotropy. When the 
local coherence, measured by $\left(\mu_{1}-\mu_{2}\right)$, is close to zero, i.e., the region corresponds to speckle pattern close to FFS carrying little tissue information, diffusion must become isotropic $\left(\mu_{1} \approx \mu_{2}=\alpha\right)$ and inversely proportional to the information content [which is also related to $\left(\mu_{1}-\mu_{2}\right)$ ]. On the other hand, when image anisotropy becomes large, which corresponds to structured tissue, image texture is rich of information about the imaged tissue and, therefore, diffusion should be very selective in both direction and strength. This idea is implemented by the above-mentioned Tukey's function, which is monotonically decreasing such that as information content increases, diffusion becomes increasingly anisotropic. A fully specular region corresponding to $\left(\mu_{1}-\mu_{2}\right)^{2}>s^{2}$ is associated with diffusion only in level set, contour, direction. Fig. 1 shows the response of the system when applied to different types of regions. In all cases, smoothing is represented as an elliptic Gaussian kernel whose radii are shown. When the region is highly specular, it is merely a one-dimensional (1-D) Gausian smoothing in the principal minimum variation direction.

The proposed NCD model has the same principal PDE described in [14]. Nevertheless, from the functional point of view, it combines three different models together; namely, isotropic linear diffusion, anisotropic diffusion, and MCM. According to speckle extent and image anisotropy, the model changes progressively from isotropic diffusion through anisotropic coherent diffusion to, finally, MCM. This structure maximally low-pass filters those parts of the image that correspond to fully developed speckle, while substantially preserving information associated with resolved-object structures [24]. Several reasons support this model formulation. First, our main objective is to produce a filter that is not only edge preserving but also coherence enhancing. This is essential to overcome the ambiguity of using the SNR alone and to further enhance tissue texture, organ surfaces, and blood vessels. Also, $\lambda_{1}$ is related to the anisotropy of the image $\left(\mu_{1}-\mu_{2}\right)$ through a monotonically decreasing function that resemble Tukey's biweight robust estimator, which preserves sharp boundaries and improves the automatic stopping of the diffusion in the gradient direction [13]. Finally, the stopping level $\mathrm{s}^{2}$ is determined experimentally corresponding to the fully structured region resulting from the imaging system and manually set by the user.

\section{Pseudobiased Diffusion}

Although the previous model has proved to be efficient in removing speckle, if prior knowledge of the appropriate time evolution needed to reach this speckle-free image is not available, the model continues to evolve to the trivial solution. This behavior is not recommended for parameter robustness. The overall time step is a very important parameter that drastically influences the final status of the filtered image. However, the behavior of a robust filtering algorithm should not be sensitive to parameter selection especially time evolution. Therefore, the flow dynamics of the diffusion process should be controlled based on both the spatial local coherence as well as the temporal distance between the original speckled image and the current evolved image [25]. Meanwhile, the added diffusion control should not increase (at least, minimally) the complexity of the numerical implementation of the process. The proposed model can be summarized in the design of the required diffusion eigenvalues.

Let

$$
\delta=1-\beta \cdot\left(\left|I\left(t_{0}\right)-I(t)\right|\right), \quad 0 \leq \beta \leq 1
$$

and

$$
\begin{aligned}
& \lambda_{1}= \begin{cases}\alpha \cdot \delta \cdot\left(1-\frac{\left(\mu_{1}-\mu_{2}\right)^{2}}{s^{2}}\right), & \text { if }\left(\mu_{1}-\mu_{2}\right)^{2} \leq s^{2} \\
0, & \text { else }\end{cases} \\
& \lambda_{2}=\alpha \cdot \delta .
\end{aligned}
$$

The distance between the original and the evolved images [represented in (19)] is continuously measured at all time instants and is embedded within the formulation of the eigenvalues to control the status of diffusion process. This modification has the effect of completely stopping the flow of the diffusion at any time and location where the deviation between the two images becomes unreasonable as will be analyzed.

The dynamics of the proposed model can be clearly understood from the eigen-analysis framework. The flow is affected by two factors; the first one is the distance between the evolved image at any time $t$ and the original image at time $t=0$ (that we call temporal distance). The second factor is the spatial local coherence or anisotropy (spatial coherence). At early time values $\left(t \approx t_{0}\right)$, the temporal distance effect is completely negligible $(\delta \approx 1)$ and the only dominant factor is that of the spatial coherence. When the local coherence, measured by $\left(\mu_{1}-\mu_{2}\right)$, is close to zero, i.e., the region corresponds to speckle pattern close to FFS carrying little tissue information, diffusion must become isotropic $\left(\mu_{1} \approx \mu_{2}=\alpha\right)$ and inversely proportional to the information content (which is also related to $\left(\mu_{1}-\mu_{2}\right)$ ). On the other hand, when image anisotropy becomes large, which corresponds to structured tissue, image texture is rich of information about the imaged tissue and, therefore, diffusion should be very selective in both direction and strength. This idea is implemented by the above-mentioned Tukey's function, which is monotonically decreasing such that as information content increases, diffusion becomes increasingly anisotropic. A fully structured region corresponding to $\left(\lambda_{1}-\lambda_{2}\right)^{2}>s^{2}$ is associated with diffusion only in contour direction.

As time evolution goes forward, the image continues to diffuse and some structures may be inevitably unsharpened. In such cases, the temporal distance increases between the original and diffused edge sharpness. The embedding of this distance in the diffusion matrix has the effect of choking extra diffusion in these locations. This effect dominates over the effect of spatial coherence and thus, limiting diffusion. With time, the unshaped edges become relatively sharper than before with respect to inter-region fluctuations [25]. Hence, the stopping criterion in this formulation can be made that the difference between the results from two consecutive iterations becomes less than a preset minimum.

\section{Discretization Scheme}

The final form of the model takes the form

$$
\frac{\partial I}{\partial t}=\operatorname{div}\left[\begin{array}{cc}
\left(\begin{array}{cc}
a & b \\
c & d
\end{array}\right) & \left(\begin{array}{l}
I_{x} \\
I_{y}
\end{array}\right) \\
\text { Diffusivity Matrix }(D) & \text { Gradient Vector }
\end{array}\right]
$$




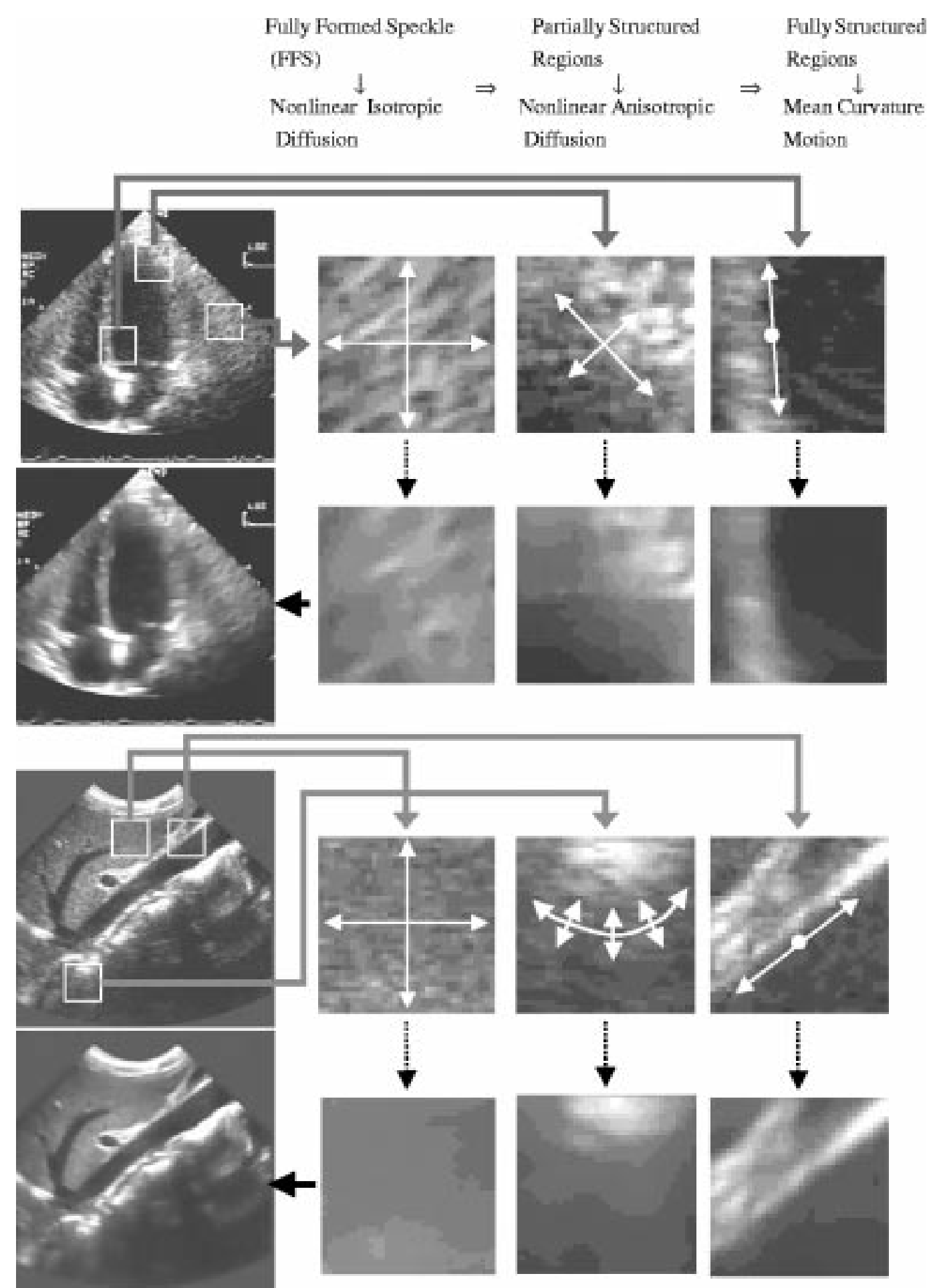

Fig. 1. The behavior of the proposed model as the speckle pattern changes. Two examples are shown with three different regions in each with the behavior of the algorithm in each region.

where $a, b, c$ and $d$ represent the entries of the diffusivity matrix in (17), which are in general functions of the local image intensity. We assume that the discrete image at hand is of size $N^{2}$. To apply the scalar model in (8), an efficient and stable discretization scheme can be used [21]. This scheme uses Euler-Backward method to obtain a system of linear equations that is stable for any time step. With a 2-D image, the resultant system involves solving a sparse matrix with number of unknown equals $N^{2}$. To overcome the problem of solving this huge matrix, the algorithm further split the works by splitting the $m$-dimensional system of equations into $m$ 1-D systems.

Due to the need for $I_{x x}, I_{y y}$, and $I_{x y}$, all eight points in the neighborhood are used in our model. As a result, several such proposed schemes cannot be applied here. Explicit scheme seems stable for only very small step $(\Delta t<1 / 4$ for the scalar diffusion) [14] and even smaller for our model. Implicit scheme is extremely complicated due to the nonlinearity and the bulky system matrix to be solved. Semi-implicit has shown uncondi- 


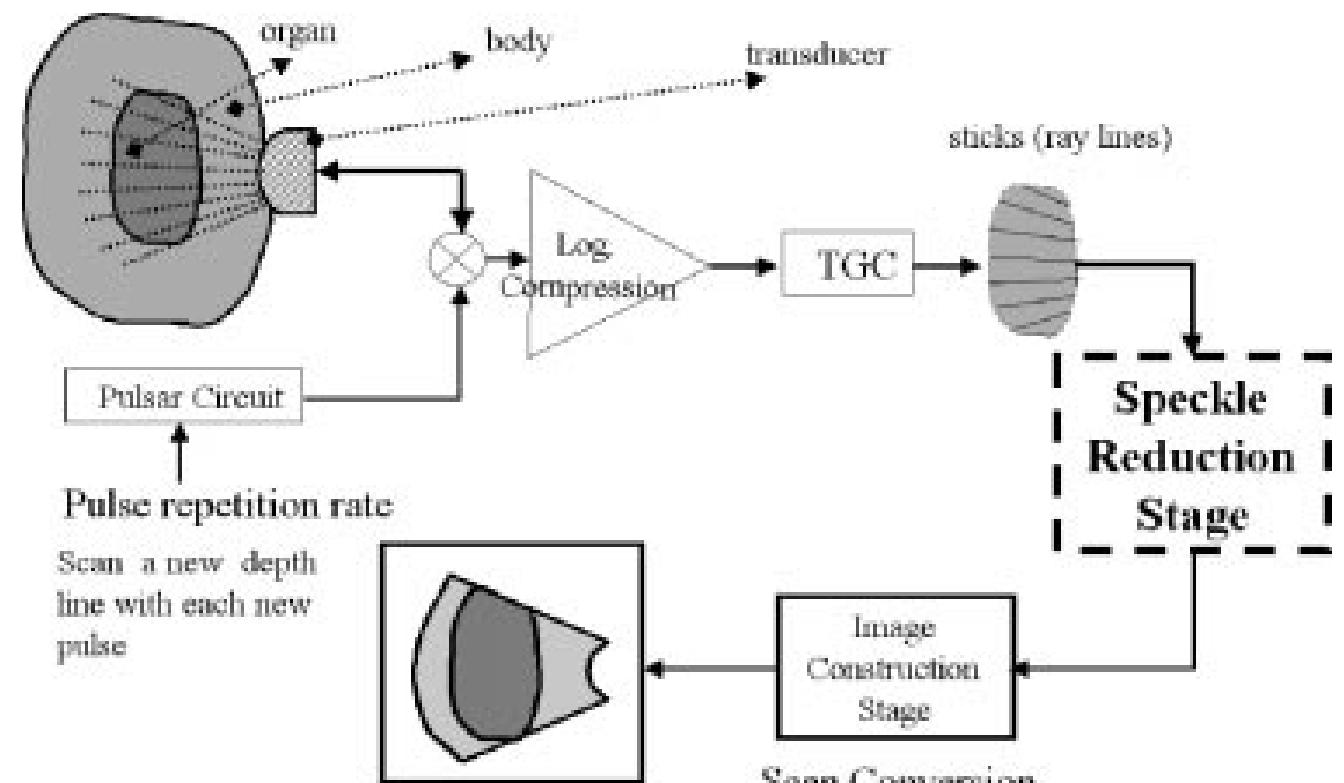

Raster Image Display

Fig. 2. Block diagram of ultrasonic convex B-mode system showing the location of the proposed speckle reduction stage. It is applied on stick lines instead of the constructed raster image.

tional stability in scalar nonlinear diffusion for any time step where the $N^{2} \times N^{2}$ pentadiagonal system matrix is divided into $2 N$ simple tridiagonal ones with each of size $N \times N$ [21]. Due to the added complexity in the coherent model, the scheme is no longer as simple. The system matrix is now pentadiagonal with fringes due to the added diagonal points. To overcome this difficulty, a hybrid scheme is proposed that combines stability and computational efficiency. The scheme can be described as shown in the equation at bottom of the page, or

$$
I_{i, j}^{t+\Delta t} \Leftarrow\left(\begin{array}{ccc}
I_{i-1, j+1}^{t} & I_{i, j+1}^{t+\Delta t} & I_{i+1, j+1}^{t} \\
I_{i-1, j}^{t+\Delta t} & I_{i, j}^{t} & I_{i+1, j}^{t+\Delta t} \\
I_{i-1, j-1}^{t} & I_{i, j-1}^{t+\Delta t} & I_{i+1, j-1}^{t}
\end{array}\right) .
$$

Here, $I_{I, j}^{t}$ stands for the pixel at location $x=i, y=j$ at time instance $t$. That is, the axial points are made backward in time and the diagonal and cross-diagonal points are made forward in time. In this scheme, splitting can be used to convert the system matrix into tridiagonal matrices, which are easily solved.

With no loss of generality, we put $\Delta t=\tau$ and $\Delta x=\Delta y=$ 1 , which is a valid assumption when dealing with images. The result takes the form

$$
\left(1-\tau \sum_{l=1}^{2} A_{l}\left(I^{k}\right)\right) I^{k+1}=J^{k+1 / 2} .
$$

Similar to [21], it can be solved using additive operator splitting scheme as

$$
I^{k+1}=\frac{1}{2} \sum_{l=1}^{2}\left(1-2 \tau A_{l}\left(I^{k}\right)\right)^{-1}\left(J^{k+1 / 2}\right)
$$

where

$$
a_{i, j}= \begin{cases}\frac{\bar{a}_{i, j}^{k}+\bar{\alpha}_{i, j \pm 1}^{k},}{2}, & j=i \pm 1, l=1 \\ \frac{\bar{d}_{i, j}^{k}+\bar{d}_{i, j \pm 1}^{k}}{2}, & j=i \pm 1, l=2 \\ a_{i, i-1}+a_{i, i+1}, & j=i \\ 0, & \text { otherwise. }\end{cases}
$$

and

$$
\begin{array}{r}
J_{j, l}^{k+1 / 2}=I_{j, l}^{k}+\frac{\Delta t}{4}\left\{\bar{c}_{j+1, l}^{k}\left(I_{j+1, l+1}^{k}-I_{j+1, l-1}^{k}\right)\right. \\
\left.-\bar{c}_{j-1, l}^{k}\left(I_{j-1, l+1}^{k}-I_{j-1, l-1}^{k}\right)\right\} \\
+\frac{\Delta t}{4}\left\{\bar{b}_{j, l+1}^{k}\left(I_{j+1, l+1}^{k}-I_{j-1, l+1}^{k}\right)\right. \\
\left.-\bar{b}_{j, l-1}^{k}\left(I_{j+1, l-1}^{k}-I_{j-1, l-1}^{k}\right)\right\} .
\end{array}
$$

\section{E. Algorithm}

An iteration of the proposed algorithm consists of the following steps.

$$
\begin{aligned}
\frac{I_{j, l}^{k+1}-I_{j, l}^{k}}{\Delta t}= & \frac{1}{\Delta y^{2}}-\left\{\left(\frac{\bar{d}_{j, l}^{k}+\bar{d}_{j, l+1}^{k}}{2}\right)\left(I_{j, l+1}^{k+1}-I_{j, l}^{k+1}\right)+\left(\frac{\bar{d}_{j, l}^{k}+\bar{d}_{j, l-1}^{k}}{2}\right)\left(I_{j, l-1}^{k+1}-I_{j, l}^{k+1}\right)\right\} \\
& +\frac{1}{\Delta x^{2}}\left\{\left(\frac{\bar{a}_{j, l}^{k}+\bar{a}_{j+1, l}^{k}}{2}\right)\left(I_{j+1, l}^{k+1}-I_{j, l}^{k+1}\right)+\left(\frac{\bar{a}_{j, l}^{k}+\bar{a}_{j-1, l}^{k}}{2}\right)\left(I_{j-1, l}^{k+1}-I_{j, l}^{k+1}\right)\right\} \\
& +\frac{1}{4 \Delta x \Delta y}\left\{\bar{c}_{j+1, l}^{k}\left(I_{j+1, l+1}^{k}-I_{j+1, l-1}^{k}\right)-\bar{c}_{j-1, l}^{k}\left(I_{j-1, l+1}^{k}-I_{j-1, l-1}^{k}\right)\right\} \\
& +\frac{1}{4 \Delta x \Delta y}\left\{\bar{b}_{j, l+1}^{k}\left(I_{j+1, l+1}^{k}-I_{j-1, l+1}^{k}\right)-\bar{b}_{j, l-1}^{k}\left(I_{j+1, l-1}^{k}-I_{j-1, l-1}^{k}\right)\right\}
\end{aligned}
$$




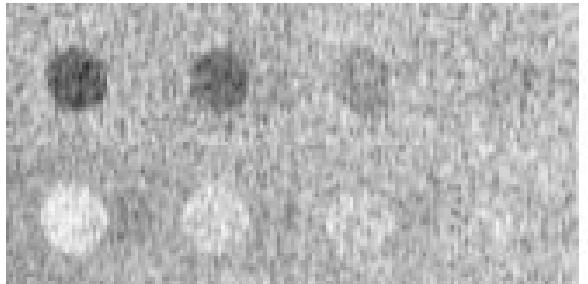

(a)

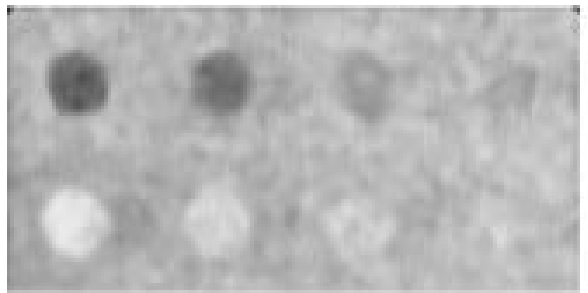

(d)

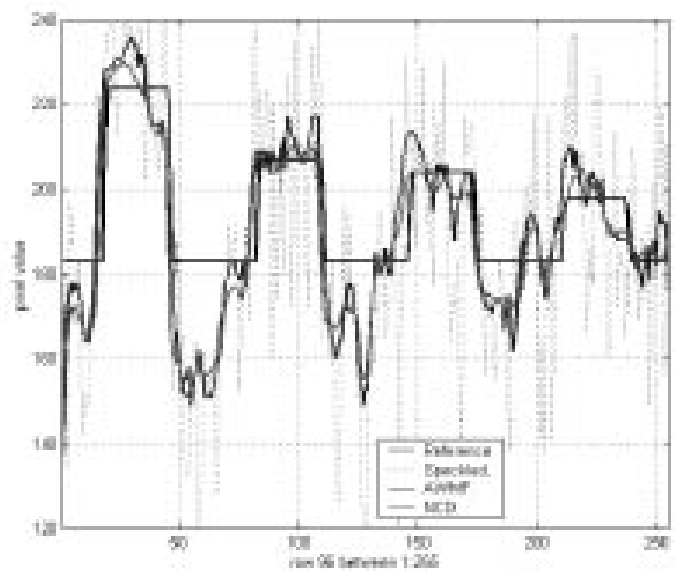

(g)

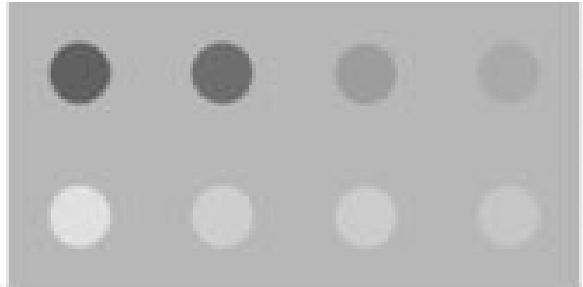

(b)

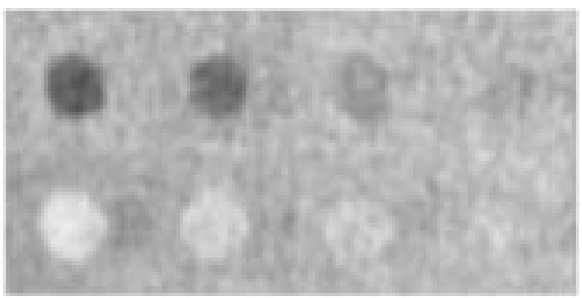

(e)

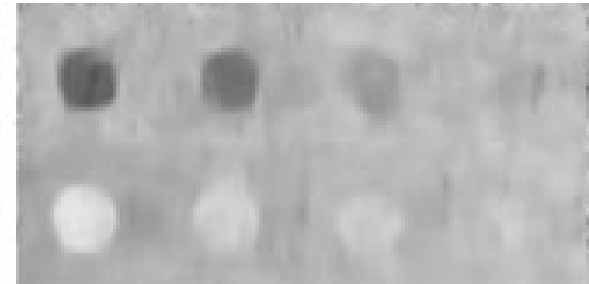

(c)

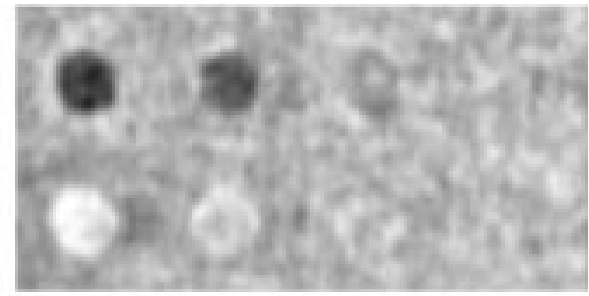

(f)
TABLE I

QuANTITATIVE EVALUATION OF THE PERFORMANCE OF APPLYING DIFFERENT TECHNIQUES TO CONTRAST PHANTOM IMAGES

\begin{tabular}{l|l}
\hline Processing Method & MSE \\
\hline AWMF & 289 \\
\hline WSCE & 271 \\
\hline WS & 132 \\
\hline NCD & 121 \\
\hline
\end{tabular}

Step 1) For each point, obtain the windowscatter matrix from (10) and the

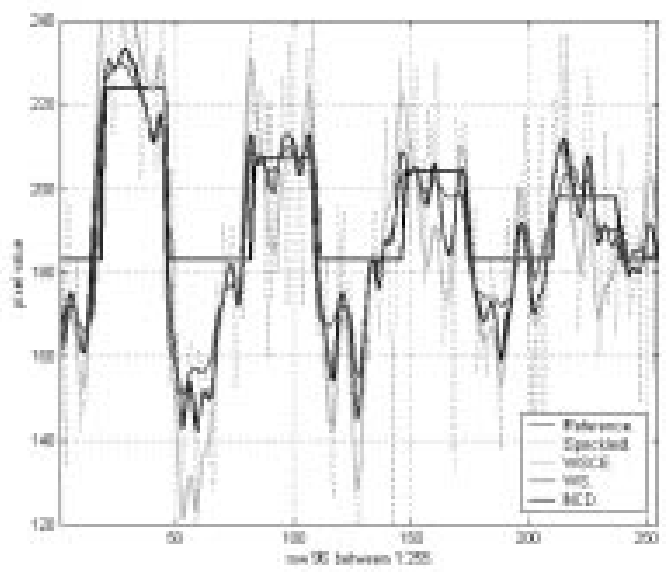

(h)

Fig. 3. Results with contrast details phantoms. (a) Original speckled image. (b) Reference image. (c)-(f) Images processed with NCD, AWMF, WS, and WSCE methods, respectively. $(\mathrm{g})$ and $(\mathrm{h})$ Profile of the evolution of a line passing through the positive contrast regions with different algorithms.

principal components and vectors from (12).

Step 2) Evaluate the diffusivity from (17) and (18) (for NCD model) or (19) and (20) (for pseudobiased NCD) based on the previously obtained scatter matrix.

Step 3) Solve the diffusion equation in (21). Time evolution of an image point is split into two parts: the Euler-Forward scheme for the time evolution involving the diagonal and cross-diagonal neighbor image points as in (25) and the Euler-Backward scheme for the time evolution involving the axial neighbor image points as in (23) and (24).

\section{F. System Implementation}

The construction of ultrasound B-mode image involves capturing the echo signal returned from tissue at the surface of 

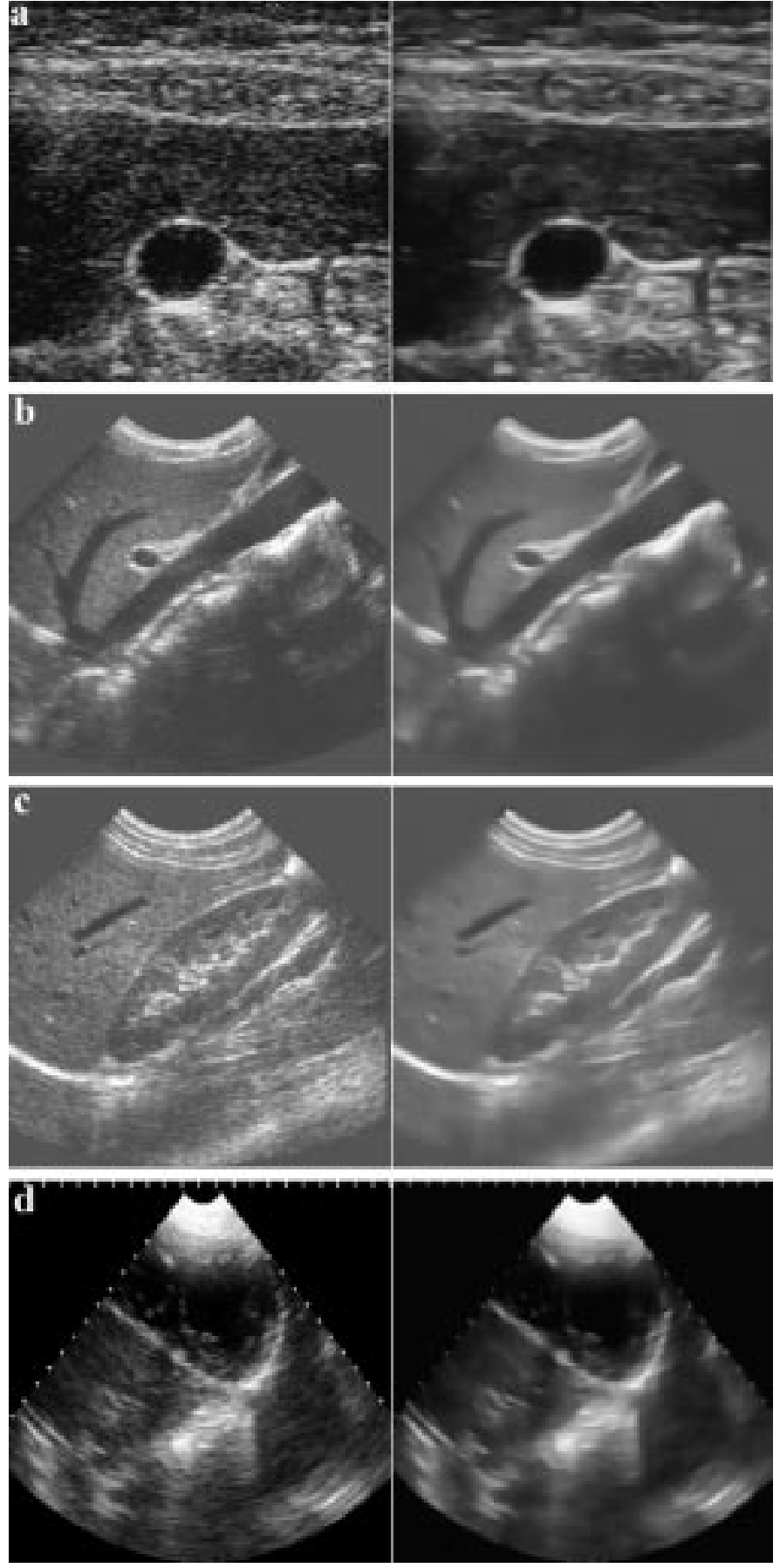

Fig. 4. The effect of the algorithm on clinical images: (a) abdomen, (b) blood vessels, (c) kidney, and (d) heart- short axis view. The number of iterations used was two, and the parameters used were $\Delta t=2, \alpha=0.9$, and $s^{2}=2$. The original image is shown to the left while the denoised image using NCD is shown to the right in each case.

piezoelectric crystal transducers. These transducers convert the ultrasonic RF mechanical wave into electrical signal. Convex ultrasound probes collect the echo from tissue in a radial form. Each group of transducers is simultaneously activated to look at a certain spatial direction from which they generate a raw line signal (stick) to be used later for raster image construction [26]. These sticks are then demodulated and logarithmically compressed to reduce their dynamic range to suit the commercial display devices. The final Cartesian image is constructed

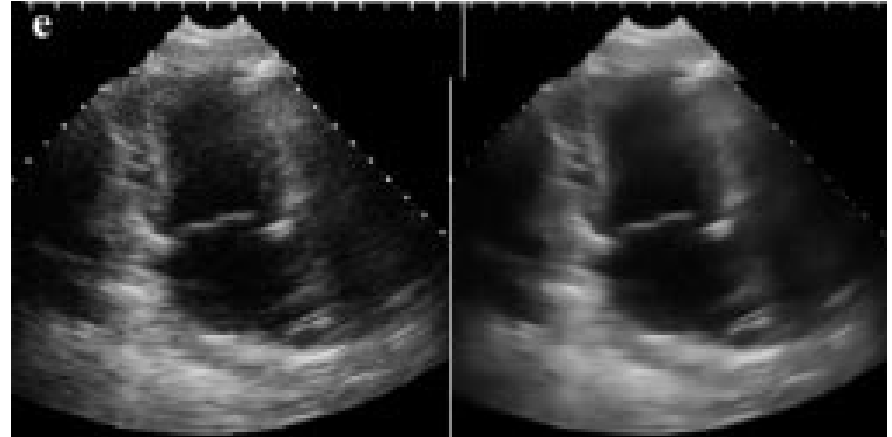

Fig. 4. (Continued.) The effect of the algorithm on clinical images. (e) Hearttwo chambers view. The number of iterations used was two, and the parameters used were $\Delta t=2, \alpha=0.9$, and $s^{2}=2$. The original image is shown to the left while the denoised image using NCD is shown to the right in each case.

from the sampled sticks in a process called scan conversion (see Fig. 2).

For the sake of efficiency and accuracy, the proposed model is applied on the ultrasound convex B-mode sampled sticks after the logarithmic compression and before constructing the final rectangular image. The machine we used forms 80 sticks or lines of 320 samples each. Hence, the original radial image size is $80 \times 320$. Since the stick coordinates are closer to radial than to the Cartesian, the model is modified simply by adjusting the gradient step in the axial direction to take into consideration the diverging pattern of the sticks. The advantage of using the sticks before scan conversion is that the small number of pixels makes the algorithm at least three times faster. Furthermore, the algorithm is now more accurate since the coordinate system of the image is the same as that of the probe. This step enables the achievement of real-time rates using the available modest computing platforms.

\section{RESULTS}

To test the performance of the proposed NCD model, we compared its results against those of one of the most used real-time models, which is the AWMF model. In addition, the results were compared with those from off-line sophisticated models such as the wavelet shrinkage (WS) model and the wavelet shrinkage coherence enhancing (WSCE) models. Experiments included images containing diverse shapes and sizes of structural details. We applied the same wavelet functions described in [17]. The images were decomposed into four levels. The high-frequency content of each level is then removed using a threshold value $T_{i}$ that is level dependant, varying linearly from $T_{\max }$ to $T_{\min }$. These bounds are functions of the noise level in the signal estimated using the standard deviation of the signal. In the WSCE technique, the same parameters as the WS technique were used but in this case, the low-frequency content of the first two levels is amplified in order to enhance the features in the spatial domain. We applied these models on ultrasound phantom and real scan-converted ultrasound clinical images. Comparison is divided into two parts. First, we compare the qualitative results from the used algorithms. Second, profiles of the effect of the tested algorithms on a details line within the images are plotted to observe the behavior of these methods. 


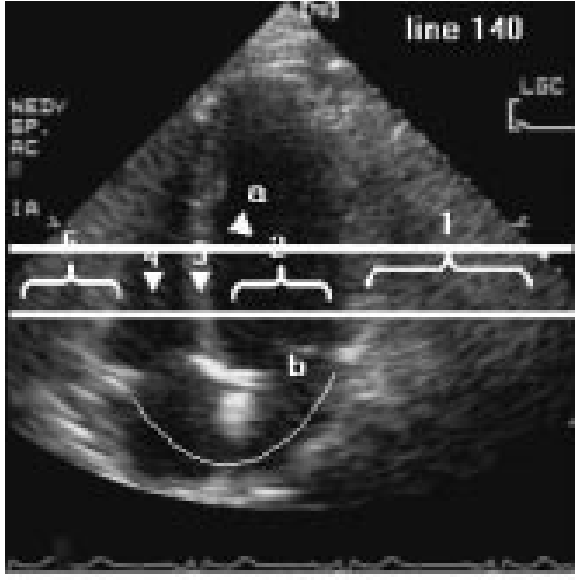

(a)

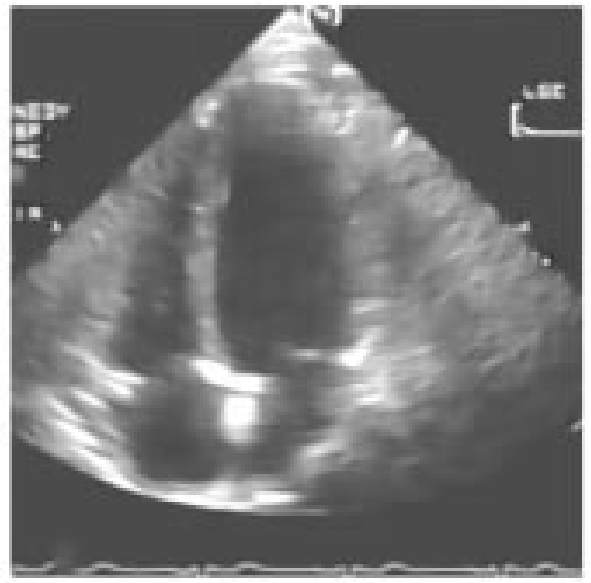

(b)

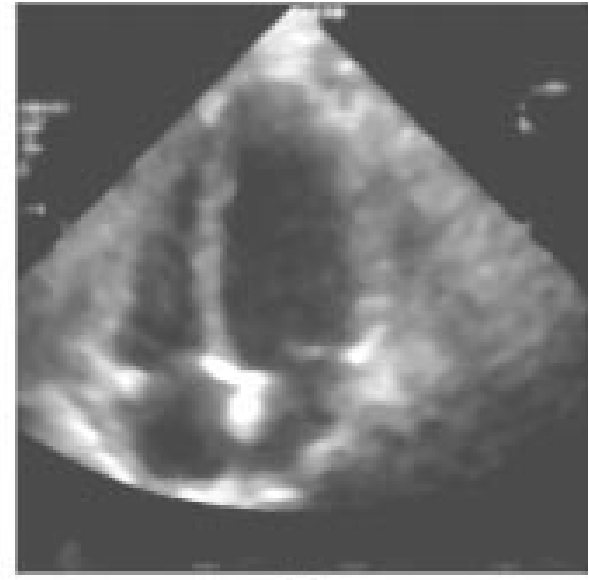

(c)

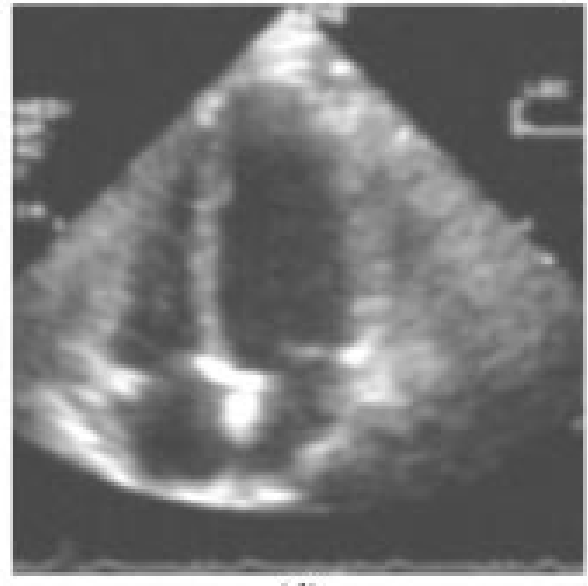

(d)

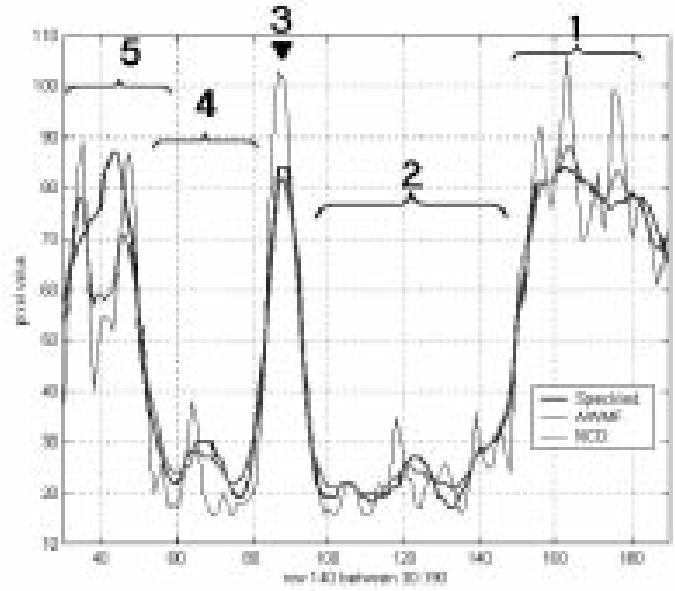

(f)

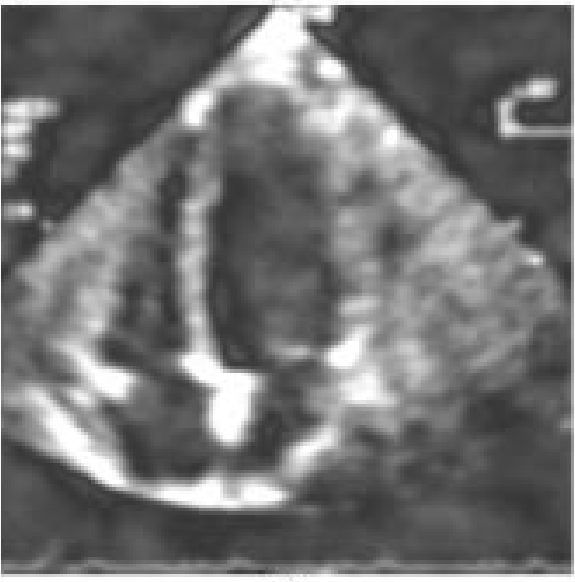

(c)

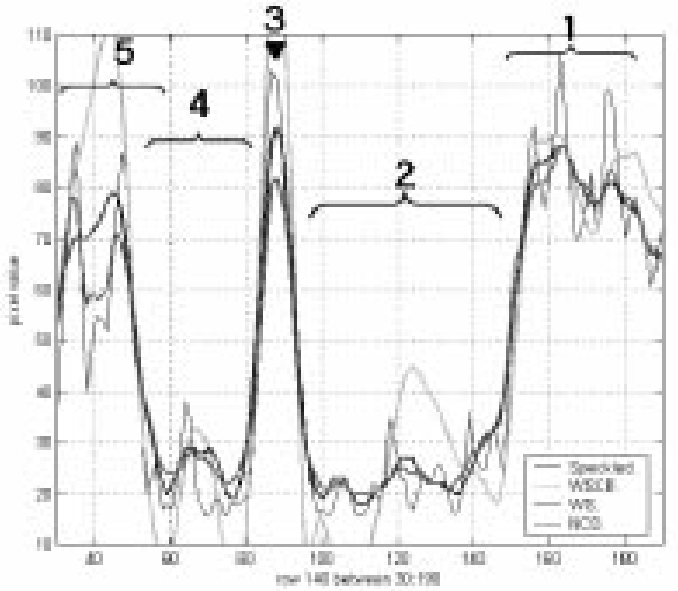

(g)

Fig. 5. The results of an ultrasound image of the heart. (a) Original speckled image with line mark superimposed. (b)-(e) Images processed with NCD, AWMF, WS, and WSCE methods, respectively. (f) and (g) Profile of the evolution of the row line labeled in (a) with different algorithms.

\section{A. Contrast Detail Phantom Data}

The four techniques were applied to enhance images of a commercial contrast detail phantom (ATS laboratories, Bridgeport, CT). This phantom was made to produce standard contrast levels from $-15 \mathrm{~dB}$ to $+12 \mathrm{~dB}$. Images were logarithmically compressed in the postprocessing stage to simulate compressed B-scan images [15]. Fig. 3 shows the results of this comparison. The phantom image has a resolution of $256 \times 128$ and consists of eight different contrast regions (four positive contrast regions and four negative contrast regions). Regions are ordered in two rows. The upper row contains negative contrast regions while the lower one contains the positive contrast regions as shown in Fig. 3(a). A reference image, Fig. 3(b), is constructed manually from the speckled image by evaluating the mean value in each region. The obtained results are shown in Fig. 3(c)-(f) corresponding to NCD, AWMF, WS, and WSCE techniques, respectively. Fig. 3(g) and (h) shows a comparison between test methods through a profile of their effect on a positive contrast 


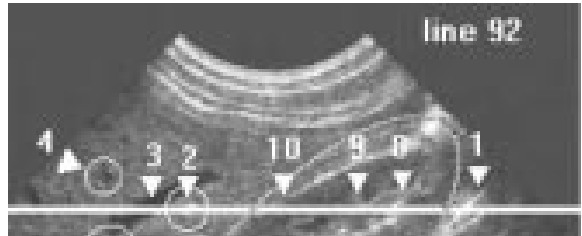

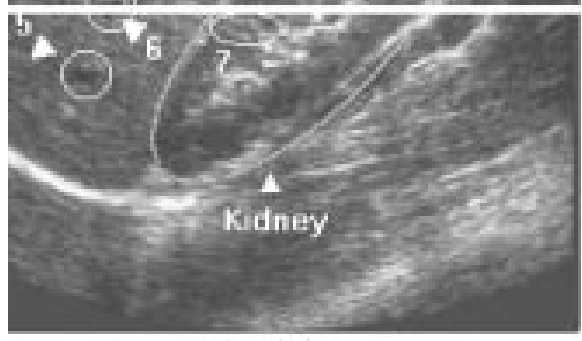

(a)

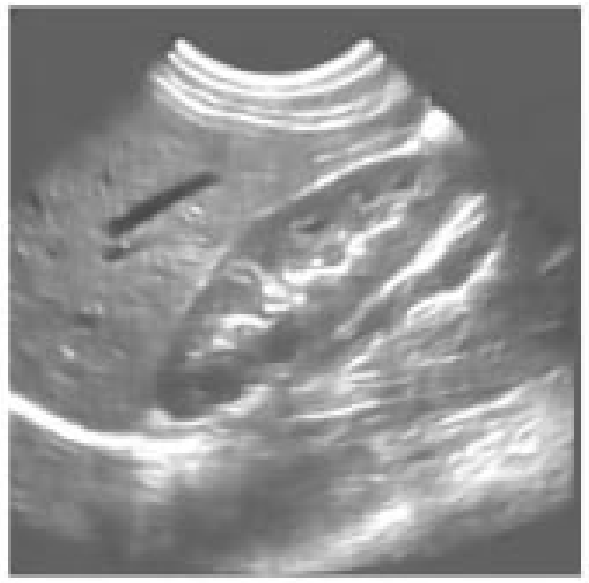

(b)

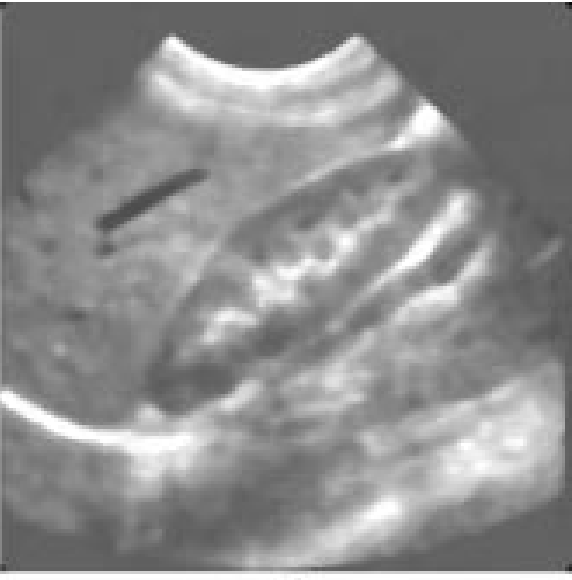

(c)

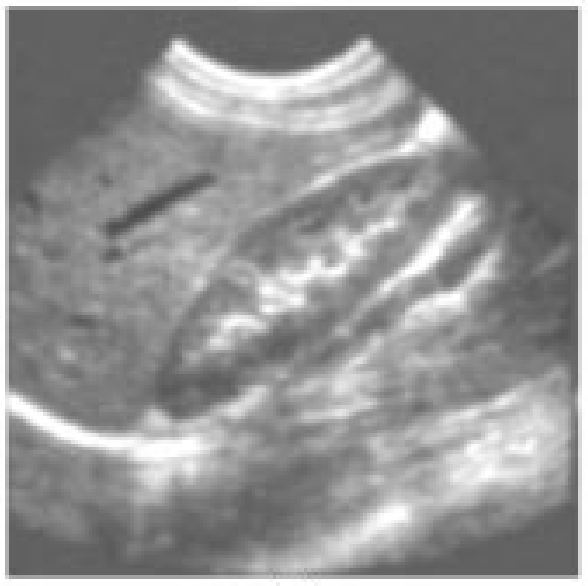

(d)

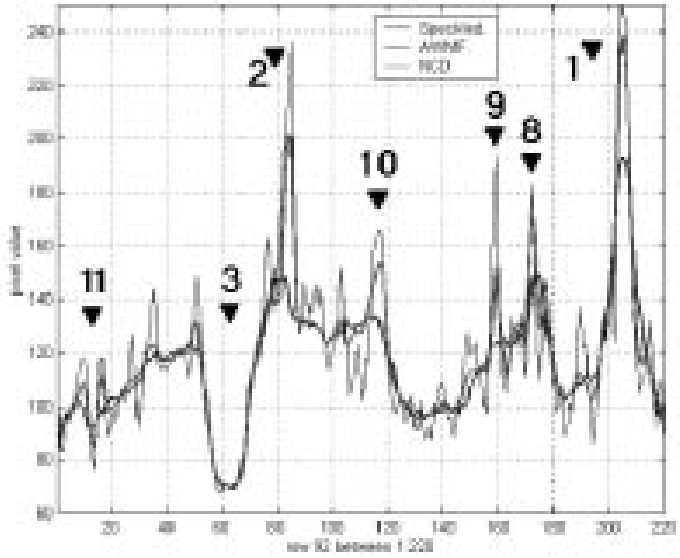

(f)

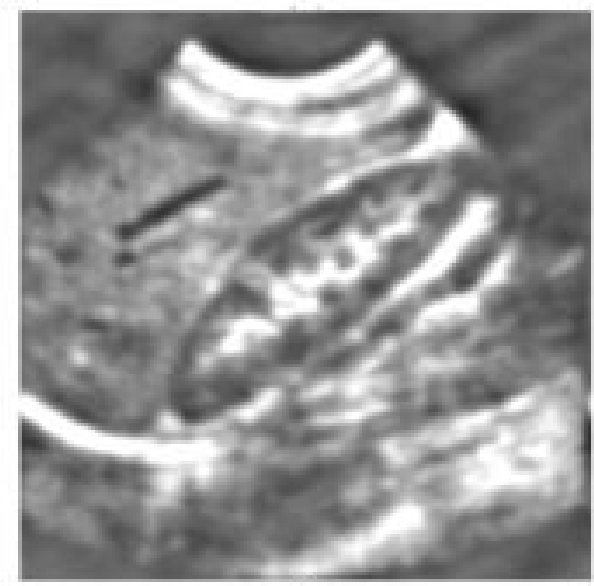

(e)

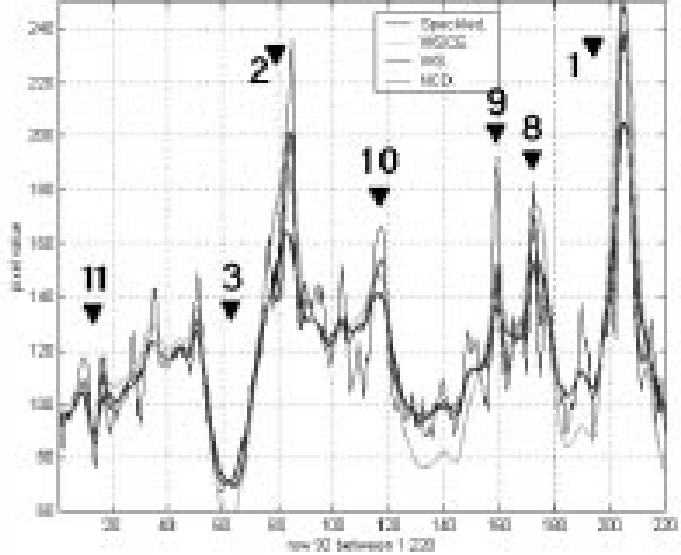

(g)

Fig. 6. The results of an ultrasound image of a kidney. (a) Original speckled image with line mark superimposed. (b)-(e) Images processed with NCD, AWMF, WS, and WSCE methods, respectively. (f) and (g) Profile of the evolution of the row line labeled in (a) with different algorithms.

line. Image results show that the proposed method looks very close to the reference image. Profile results show that although all methods, in general, preserved the locality of boundaries, the NCD model also stayed close to the reference intraregion values with minimal variations within each region.

To evaluate the results in a quantitative manner, the mean-square error (MSE) of the different techniques is listed in Table I. As can be observed, the result of the NCD model is superior to other techniques.

\section{B. Clinical Ultrasound Imaging Data}

Phantom images proved the efficiency of the proposed model in separating Rayleigh distributed noise while preserving both the locality of edges and the intraregion mean value. With the simplicity of phantom structures (boundaries only), the behavior of NCD is similar to MCM model. In the following, we present a comparison using three different clinical ultrasound images corresponding to images of the heart, kidney, and liver images. 

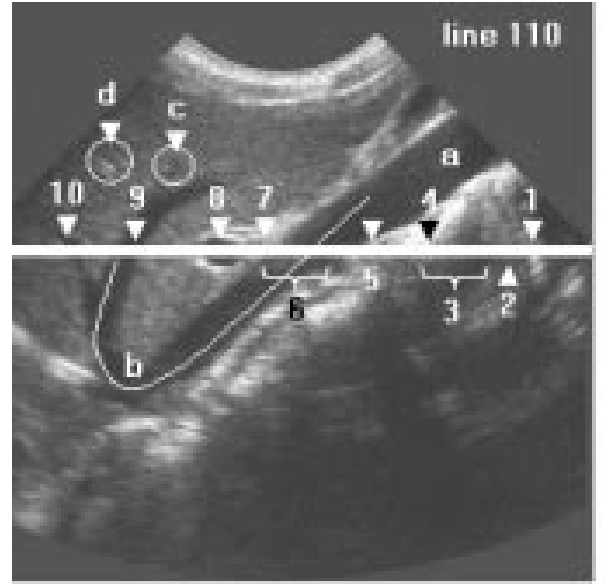

(a)

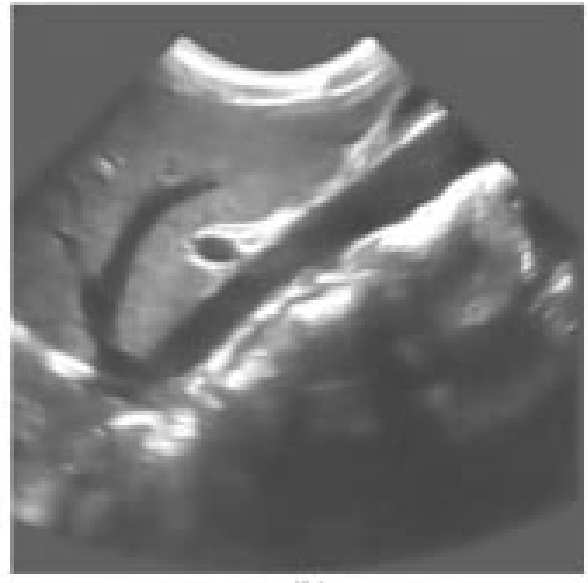

(b)

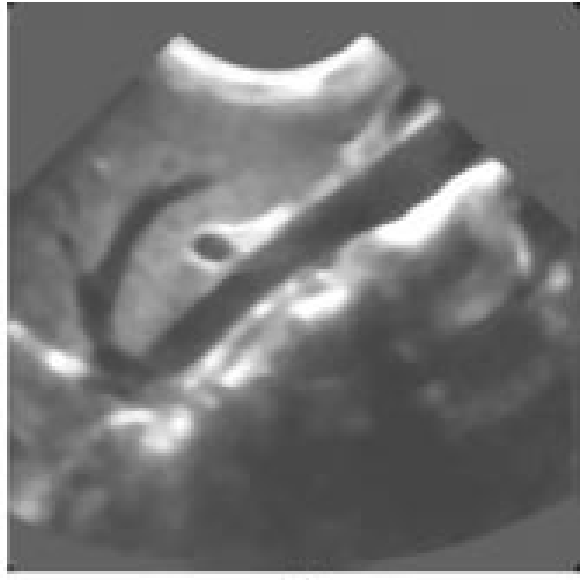

(c)

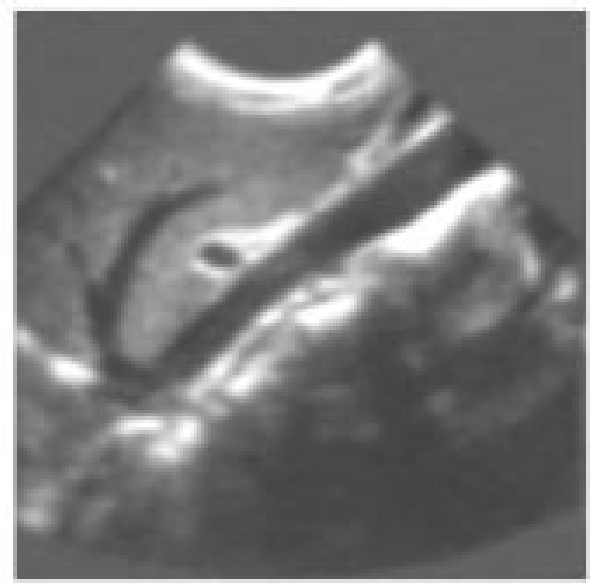

(d)

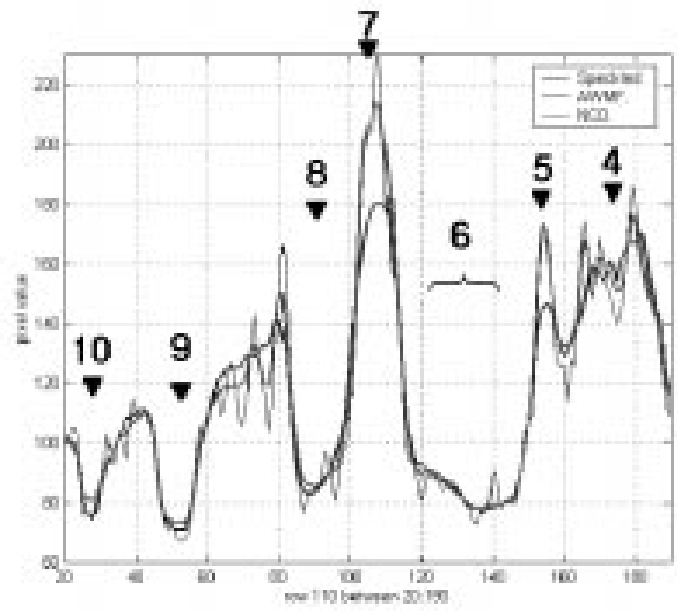

(f)

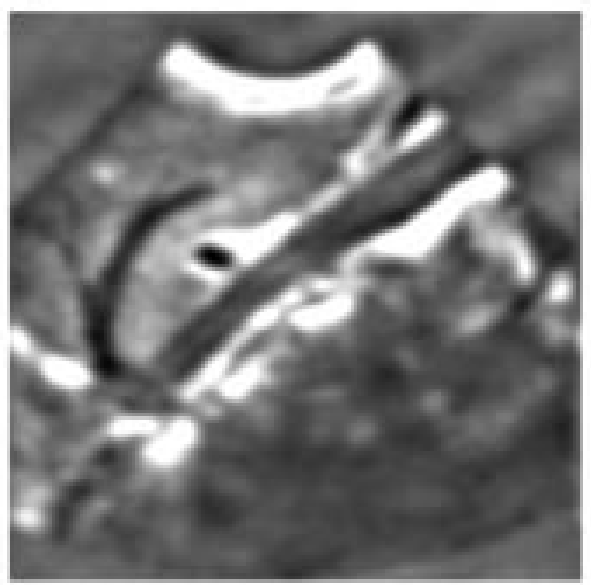

(c)

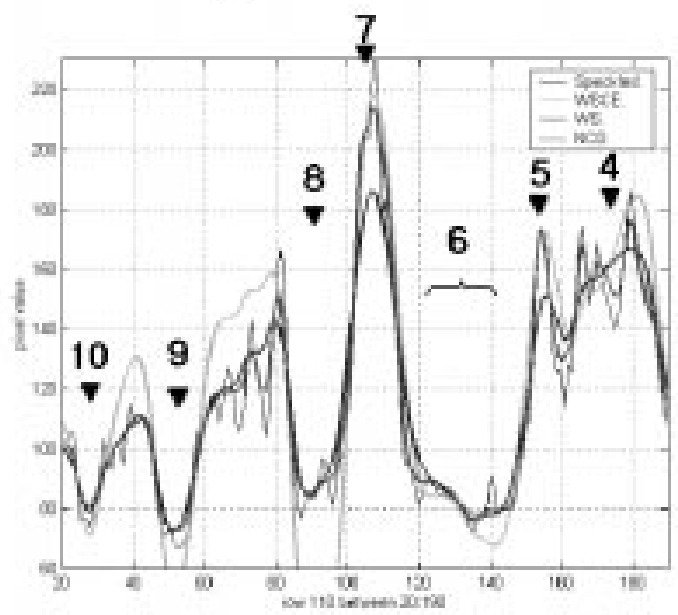

(g)

Fig. 7. The results of an ultrasound image of liver texture and blood vessels. (a) Original speckled image with line mark superimposed. (b)-(e) Images processed with NCD, AWMF, WS, and WSCE methods, respectively. (f) and (g) Profile of the evolution of the row line labeled in (b) with different algorithms.

These images exhibit different scales of information and should be useful to differentiate between the performances of the four techniques. Sample results for applying the proposed method on clinical images are shown in Fig. 4.

Case 1) Heart image

Results of this experiment are shown in Fig. 5. The proposed model succeeded in preserving the locality of heart boundaries while maximally smoothing both cavities and muscular tissue. WS and AWMF could not remove the speckle pattern inside heart cavity and boundaries were blurred. The profile line shows that the cavities (marks 2 and 4) are maximally smoothed while structures (marked as 5) are best preserved by NCD. 

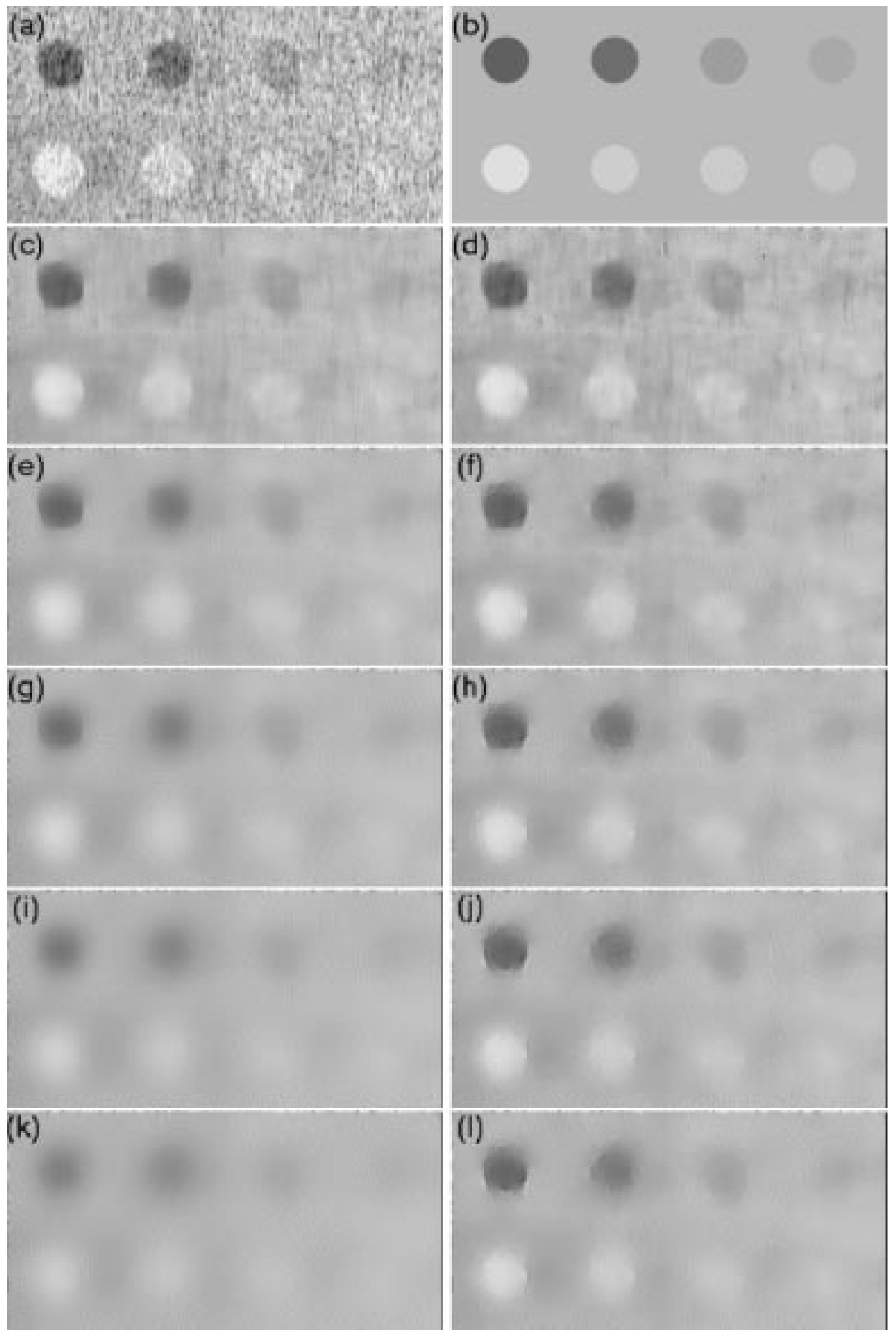

Fig. 8. Phantom results. (a) Speckled image. (b) Reference image. (c), (e), (g), (i), and (k) Evolution of unbiased diffusion after four, eight, 12, 16, and 20 iterations, respectively. (d), (f), (h), (j), and (l) Evolution of pseudobiased diffusion after four, eight, 12, 16, and 20 iterations, respectively.

\section{Case 2) Kidney image}

The results of this experiment are shown in Fig. 6. NCD succeeded to achieve speckle smoothing while preserving the structures and without any noticeable dilation. It can be seen that the NCD model compares favorably with the other three models.

\section{Case 3) Liver image}

The results of this experiment are shown in Fig. 7. This case contains both small and large details. From the evolution lines, we can see the high sensitivity of the new NCD model to small details and none of the marked details has been distorted. 

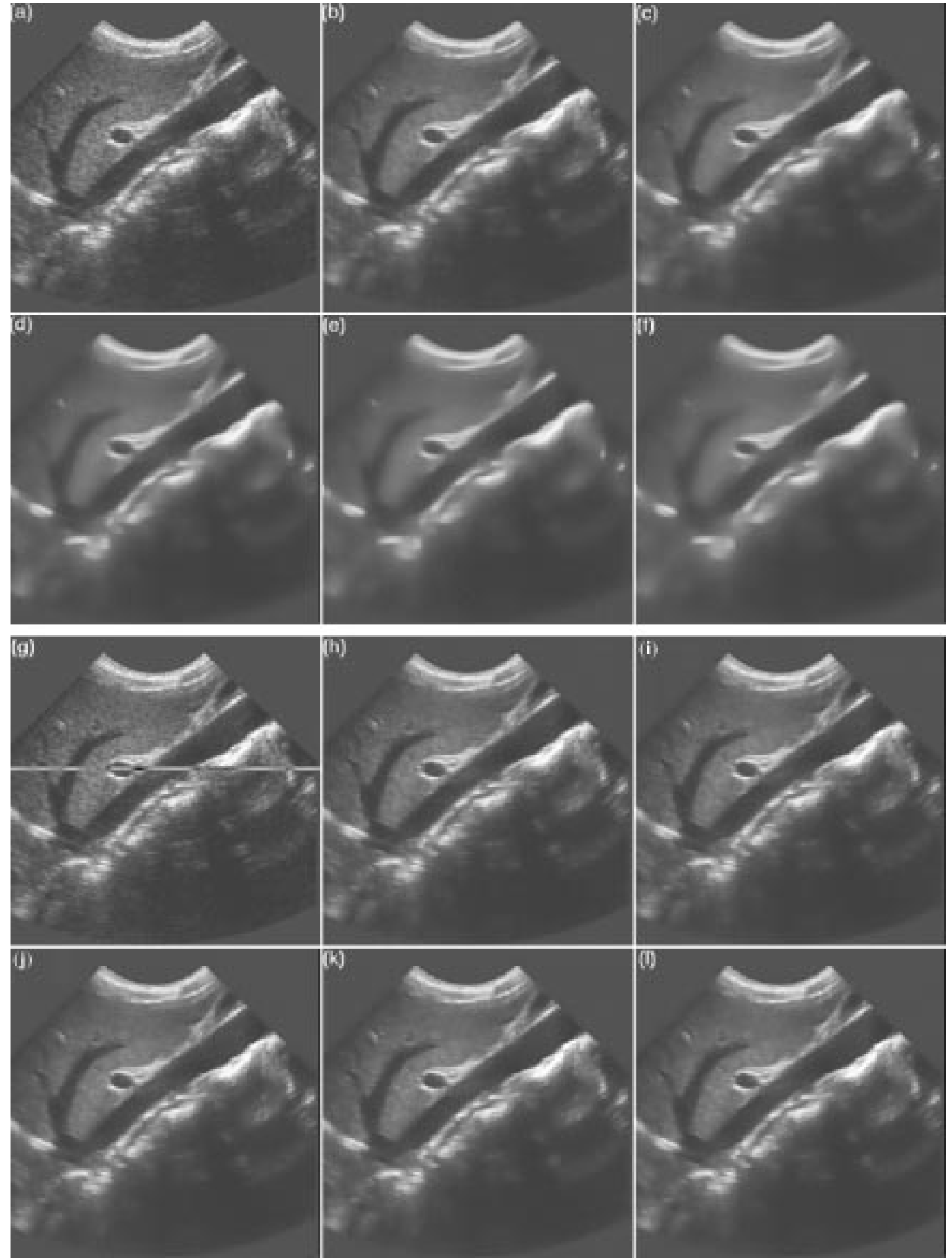

Fig. 9. The evolution of a real ultrasound image with both models. (a) Original speckled image. (b)-(f) The evolution with the unbiased diffusion after two, four, six, eight, and ten iterations respectively. (g) Speckled image with a detail line position indicated. (h)-(l) Evolution with the pseudobiased model after two, four, six, eight, and ten iterations, respectively.

\section{Pseudobiased Model Results}

The NCD model was applied with the eigenvalues as in (18) to represent the unbiased diffusion and with the eigenvalues of (20) to represent the proposed pseudobiased diffusion. Both models utilized the same values for the common algorithm parameters. To clarify flow improvement by the proposed model, we did the phantom experiment with relaxed parameters. Both models were applied to filter phantom images for 20 iterations with a time step $\Delta t=3$ per iteration, $\alpha=1, s=70$, and $\beta=0.05$ 

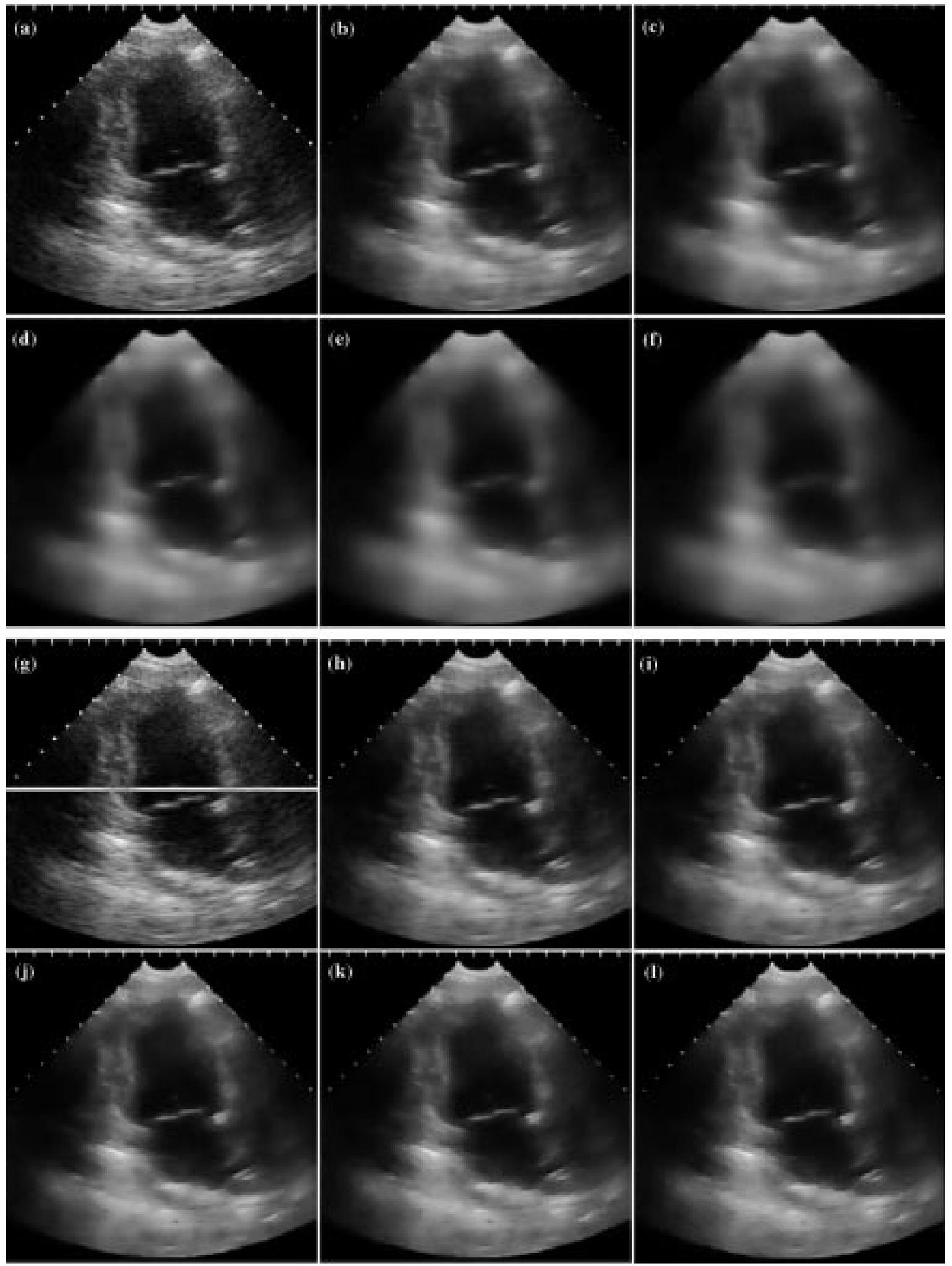

Fig. 10. The evolution of a real ultrasound image of the heart with both models. (a) Original speckled image. (b)-(f) Evolution with the unbiased diffusion after two, four, six, eight, and ten iterations respectively. (g) Speckled image with a detail line position indicated. (h)-(l) Evolution with the pseudobiased model after two, four, six, eight, and ten iterations, respectively.

in the pseudobiased model. Fig. 8 shows the evolution of both models with successive iterations. Fig. 11(a) and (b) shows the evolution of a line passing through different positive contrast regions with both models.
The two algorithms were applied to filter real ultrasound images of a blood vessel and the heart chambers. The size of both images was $256 \times 256$. In this experiment, we employed parameter values that are more conservative. Both models share 




Fig. 11. Plot of reference lines from images of previous figures to observe the evolution with unbiased diffusion (left). The evolution with pseudobiased diffusion (right). (upper panels) A positive contrast line from Fig. 8. (middle panels) A line whose position is marked in Fig. 9. (lower panels) A line whose position is marked in Fig. 10.

the same parameters for ten iterations with a time step $\Delta t=2$ per iteration, $\alpha=1, s=20$, and $\beta=0.2$ in the pseudobiased model. Figs. 9 and 10 show the results obtained from the two images. Fig. 11(c)-(f) presents the evolution of two lines through the images.
From the above results, we observe that the edges of both phantom and vessel images have been restored better with the pseudobiased model even with excessive iterations. The unbiased model is still iteration-dependant and thus continues toward the trivial single gray level solution. Edge preservation and 
iteration-robustness of the proposed model come at only minimal extra complexity in the implementation of the model. Both unbiased and pseudobiased models require almost the same time per iteration. Since the modification was merely in the determination of the diffusion components, the proposed models share the same simplicity of discretization. Only two additions and two multiplication operations are needed per pixel for embedding of the distance component of (19) Considering the overall complexity, these additional operations can be neglected.

\section{DISCUSSION}

From the experiments above, we notice that wavelet-based methods require converting the image to the Cartesian space. This contributes to extra computational time and also reduces solution accuracy. In addition, the AWMF model does not faithfully consider the 2-D nature of the image. On the other hand, the MCM method was designed to enhance piecewise constant images, which are not generally common in ultrasound imaging. Moreover, this method is noise-sensitive due to the use of second order derivative in estimating the direction of mean curvature, which can drastically degrade the results. The proposed model mimics MCM only near borders and highly specular regions where MCM is an optimal model.

The results suggest that the new technique has a large potential in assisting segmentation techniques and automated area/volume calculation methods. Examples of these are the area calculations of heart chambers and urinary bladder. Moreover, the implementation of the new NCD model enabled a computational rate of one image per $50 \mathrm{~ms}$ (computed as $16 \mathrm{~ms} /$ iteration for an average of three iterations) on a modest computational platform consisting of a PC with PII $366-\mathrm{MHz}$ microprocessor. This shows the possibility of real-time use in commercial ultrasound imaging systems given the much higher computational speeds attainable with current PC technology. Moreover, the selection of the parameters of the iteration was observed to be robust for different types of images.

\section{CONCLUSIONS}

A new nonlinear coherent diffusion model was proposed to reduce ultrasound speckle while preserving the appearance of structured regions and organ surfaces. The new technique has the advantages of robust parameter selection, speed of computation and preservation of texture and organ surfaces. The new technique has a large potential in real-time ultrasound imaging enhancement and in assisting automated segmentation/calculation techniques.

\section{ACKNOWLEDGMENT}

The authors would like to thank Tetrad Corp. for providing some of the data used in this work.

\section{REFERENCES}

[1] C. B. Burkhardt, "Speckle in ultrasound B-mode scans," IEEE Trans. Sonics Ultrason., vol. SU-25, no. 1, pp. 1-6, Jan. 1978.

[2] R. F. Wagner and M. F. Insana, "Analysis of ultrasound image texture via generalized Rician statistics,” Proc. SPIE, vol. 556, pp. 153-159, 1985.
[3] J. C. Bamber and C. Daft, "Adaptive filtering for reduction of speckle in ultrasound pulse-echo images," Ultrasonics, pp. 41-44, Jan. 1986.

[4] J. C. Bamber and G. Cook-Martin, "Texture analysis and speckle reduction in medical echography," Proc. SPIE, vol. 768, pp. 120-127, 1987.

[5] D. C. Crawford, D. S. Bell, and J. C. Bamber, "Implementation of ultrasound speckle filters for clinical trial," in Proc. IEEE Ultrason. Symp., 1990, pp. 1589-1592

[6] J. C. Bamber and J. V. Philps, "Real-time implementation of coherent speckle suppression in B-scan images," Ultrasonics, vol. 29, pp. 218-224, May 1991.

[7] D. C. Crowford, D. S. Bell, and J. C. Bamber, "Compensation for the signal processing characteristics of ultrasound B-mode scanners in adaptive speckle reduction," Ultrasound Med. Biol., vol. 19, pp. 469-485, 1993.

[8] T. Loupas, W. N. Mcdicken, and P. L. Allan, "An adaptive weighted median filter for speckle suppression in medical ultrasonic images," IEEE Trans. Circuits Syst., vol. 36, pp. 129-135, Jan. 1989.

[9] P. Perona and J. Malik, "Scale space and edge detection using anisotropic diffusion," IEEE Trans. Pattern Anal. Machine Intell., vol. 12, pp. 629-639, July 1990.

[10] S. Osher and L. Rudin, "Shock and other nonlinear filtering applied to image processing," in Proc. SPIE Appl. Digital Imag. Proc. XIV, vol. 1567, 1991, pp. 16-22.

[11] L. Alvarez, P. L. Lions, and J. M. Morel, "Image selective smoothing and edge detection by nonlinear diffusion," SIAM J. Num. Anal., vol. 29, pp. 845-866, 1992.

[12] A. Yezzi, "Modified curvature motion for image smoothing and enhancement," IEEE Trans. Imag. Processing, vol. 7, pp. 345-352, Mar. 1998.

[13] M. J. Black, G. Sapiro, D. H. Marimont, and D. Heeger, "Robust anisotropic diffusion," IEEE Trans. Imag. Processing, vol. 7, pp. 412-432, Mar. 1998.

[14] J. Weickert, "Mutiscale texture enhancement," in Lecture Notes in. Computer Science, ser. Berlin, 1995, vol. 970, Computer Analysis of Images and Patterns, pp. 230-237.

[15] V. Dutt, "Statistical analysis of ultrasound echo envelope," Ph.D. dissertation, Mayo Graduate School, Rochester, MN, 1995.

[16] J. Lu and D. M. Healy Jr, "Contrast enhancement of medical images using multiscale edge representation," Proc. SPIE, vol. 2242, pp. 711-719, 1996.

[17] X. Zong, A. F. Laine, and E. A. Geiser, "Speckle reduction and contrast enhancement of echocardiograms via multiscale nonlinear processing," IEEE Trans. Med. Imag., vol. 17, Aug. 1998.

[18] A. F. Laine, S. Schuler, J. Fan, and W. Huda, "Mammographic feature enhancement by multiscale analysis," IEEE Trans. Med. Imag., vol. 13, pp. 725-740, Apr. 1994.

[19] A. Aldroubi and M. Unser, Wavelets in Medicine and Biology. Boca Raton, FL: CRC, 1996.

[20] Y. Xu et al., "Wavelet transform domain filters: A spatially selective noise filtration technique," IEEE Trans. Imag. Processing, vol. 3, no. 6, pp. 747-758, Nov. 1994.

[21] J. Weickert et al., "Efficient and reliable schemes for nonlinear diffusion filtering," IEEE Trans. Imag. Processing, vol. 7, pp. 398-410, Mar. 1998.

[22] D. L. Donoho, "De-noising by soft-thresholding," IEEE Trans. Inform Theory, vol. 41, pp. 613-627, May 1995.

[23] X. Hao, S. Gao, and X. Gao, "A novel multiscale nonlinear thresholding method for ultrasonic speckle suppressing," IEEE Trans. Med. Imag., vol. 18, pp. 787-794, Sept. 1999.

[24] K. Z. Abd-Elmoniem, Y. M. Kadah, and A. M. Youssef, "Real time adaptive ultrasound speckle reduction and coherence enhancement," presented at the Int. Conf. Imag. Processing (ICIP' 2000), Vancouver, Canada.

[25] K. Z. Abd-Elmoniem, A. M. Youssef, and Y. M. Kadah, "Pseudo-biased coherent diffusion for robust real-time speckle reduction," in Proc. SPIE Med. Imag., vol. 4322, 2001, pp. 776-786.

[26] D. A. Christensen, Ultrasonic Bioinstrumentation. New York: Wiley, 1988.

[27] A. Achim, A. Bezerianos, and P. Tsakalides, "Novel Baysian multiscale method for speckle removal in medical ultrasound images," IEEE Trans. Med. Imag., vol. 20, pp. 772-783, Aug. 2001.

[28] R. A. Carmona and S. Zhong, "Adaptive smoothing respecting feature directions," IEEE Trans. Imag. Processing, vol. 7, pp. 353-8, Mar. 1998.

[29] P. M. Shankar, "A general statistical model for ultrasonic backscattering from tissues," IEEE Trans. Ultrason. Ferroelect. Freq. Contr, vol. 47, pp. 727-736, Mar. 2000. 


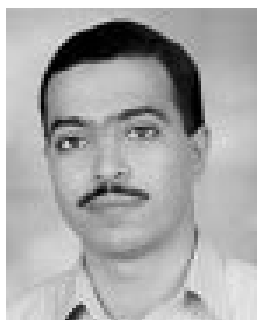

Khaled Z. Abd-Elmoniem ( $\mathrm{S}^{\prime} 02$ ) received the B.Sc. and the M.Sc. degrees in biomedical engineering from Cairo University, Cairo, Egypt, in 1997 and 2000, respectively. He is currently working toward the Ph.D. degree in electrical engineering at Johns Hopkins University, Baltimore, MD.

He worked as a Teaching Assistant in the Department of Biomedical Engineering, Cairo University, from 1997 to 2001. He joined the ultrasound project in the R\&D department of the International Electronics from 1999 to 2001. His research interests include multidimensional signal processing and medical imaging.

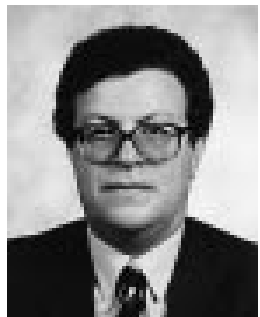

Abou-Bakr M. Youssef was born in Cairo Egypt 1951. He graduated from Cairo University-Faculty of Engineering-Electrical Engineering \& Communication Department with Distinction with Honor in 1974. Then, he joined the Faculty of Medicine and worked in parallel on engineering research and received M.S.E.E. degree in 1978, and the Ph.D. degree in biomedical engineering (MIT fellow) in 1982-Cairo University. While continuing his engineering career, he was also able to graduate from the Medical School, Cairo University 1980, and earned the $\mathrm{MBBH}$, Distinction with Honors, and the foreign equivalent, ECFMG, in 1981. In 1984, he received the M.S. degree in radiology from Cairo University, and the MD degree from Heildelberg University, Heildelberg, Germany, in 1989. He conducted medical research in the German cancer research center (DKFZ) from 1987-1993.

He contributed to the establishment of the Biomedical Engineering Department at Cairo University in 1977, developed courses of biomedical equipment and clinical engineering as well as medical imaging, and joined in the establishment of the Technology Transfer Focus in Medical School-Cairo University, in cooperation with the National Science Foundation in 1978-1980. He gained clinical experience in his research and educational life span by working as the Head of the Ultrasound Department in major hospitals in Cairo as well as within private clinics. In 1990-1992, he participated in the Health Care Development Plan, in cooperation with the Egyptian Scientific Research Academy, Cairo. Many of his researches were published in international and local scientific magazines.

Dr. Youssef was registered as a Diagnostic Medical Sonographer RDMS in 1995. As a member of the American Institute of Ultrasonography in Medicine (AIUM), in 1997 he was elected as a Senior Member. He established the Biomedical Division of International Electronics, as a practical and technical service provider for medical industry and health care community in 1991. He was appointed CEO for International Electronic Company Biomedical group, Cairo. He participated with the Information \& Decision Support Center-Egyptian Council of Ministers, in the release of medical software and expert systems in 1994. He was awarded from the government twice for the scientific research-Cairo University 1998, and the national award for advanced technological sciences-2001.

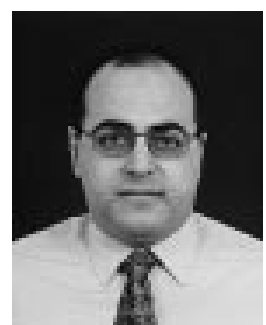

Yasser M. Kadah (M'01) was born in Giza, Egypt, in 1967. He received the B.Sc. and M.Sc. degrees in biomedical engineering from Cairo University, Cairo, Egypt, in 1989 and 1992, respectively. He received the Ph.D. in biomedical engineering from the University of Minnesota, Twin Cities, in 1997.

$\mathrm{He}$ worked as a Research Assistant with the Department of Radiology, University of Minnesota Medical School in 1996-1997 and as a Post-doctoral Fellow at the Center of Magnetic Resonance Research at the University of Minnesota in 1998. He has been an Assistant Professor of Biomedical Engineering at Cairo University since 1998 . He also worked during the same period as the Director of Research and Development at IBE technologies, Giza, Egypt. His research interests include medical imaging and, in particular, MRI and ultrasound, and multidimensional signal processing for biomedical applications.

Dr. Kadah has received several awards and recognitions including the record for highest undergraduate GPA in the department of Biomedical Engineering at Cairo University and the IDB Merit Scholarship (1993-1996), as well as the Zaviosky Award from the International Society of Magnetic Resonance in Imaging (2002). His biography was selected to appear in Marquis Who's Who in the World (2002). He is an active member of the ISMRM and SPIE. 